\title{
Star formation in Perseus
}

\section{Outflows $\mathbf{s}^{\star, \star \star}$}

\author{
J. Hatchell ${ }^{1}$, G. A. Fuller ${ }^{2}$, and J. S. Richer ${ }^{3}$ \\ 1 School of Physics, University of Exeter, Stocker Road, Exeter EX4 4QL, UK \\ e-mail: hatchell@astro.ex.ac.uk \\ 2 School of Physics and Astronomy, University of Manchester, PO Box 88, Manchester M60 1QD, UK \\ 3 Cavendish Laboratory, Cambridge CB3 OHE, UK
}

Received 28 September 2006 / Accepted 11 June 2007

\begin{abstract}
Context. We present a search for outflows towards 51 submillimetre cores in the Perseus molecular cloud.

Aims. Our first objective is to identify the protostellar population through the detection of molecular outflows. Our second aim is to consistently derive outflow properties from a large homogeneous dataset within one molecular cloud in order to investigate further the mass dependence and time evolution of protostellar mass loss.

Methods. We used the James Clerk Maxwell Telescope to map $2^{\prime} \times 2^{\prime}$ regions around each core in ${ }^{12} \mathrm{CO} 3-2$. Where molecular outflows were detected we derived momentum fluxes.

Results. Of the 51 cores, 37 show broad linewings indicative of molecular outflows. In 13 cases, the linewings could be due to confusion with neighbouring flows but 9 of those sources also have near-infrared detections confirming their protostellar nature. The total fraction of protostars in our sample is $65 \%$. All but four outflow detections are confirmed as protostellar by Spitzer IR detections and only one Spitzer source has no outflow, showing that outflow maps at this sensitivity are equally good at identifying protostars as Spitzer. Outflow momentum flux correlates both with source luminosity and with core mass but there is considerable scatter even within this one cloud despite the homogeneous dataset. We fail to confirm the result of Bontemps et al. (1996) that Class I sources show lower momentum fluxes on average than Class 0 sources, with a KS test showing a significant probability that the momentum fluxes for both Class 0s and Class Is are drawn from the same distribution.

Conclusions. We find that outflow power may not show a simple decline between the Class 0 to Class I stages. Our sample includes low momentum flux, low-luminosity Class 0 sources, possibly at a very early evolutionary stage. If the only mass loss from the core were due to outflows, cores would last for $10^{5}-10^{8}$ years, longer than current estimates of $1.5-4 \times 10^{5}$ years for the mean lifetime for the embedded phase. Additional mechanisms for removing mass from protostellar cores may be necessary.
\end{abstract}

Key words. submillimeter - stars: formation - stars: evolution - ISM: jets and outflows

\section{Introduction}

Submillimetre (submm) continuum surveys of the Perseus molecular cloud, such as our SCUBA survey, (Hatchell et al. 2005, hereafter Paper I, see also Kirk et al. 2006), and the similar $1300 \mu \mathrm{m}$ survey with Bolocam (Enoch et al. 2006), have identified over 100 submm cores. These cores could be either protostellar or starless in nature: protostellar cores can be identified by radio or infrared detection of a central source, or evidence for a protostellar outflow. In a sister paper (Hatchell et al. 2007, hereafter Paper II) we classify the protostellar cores on the basis of their spectral energy distributions, including the new data from Spitzer (Evans et al. 2003; Jørgensen et al. 2007). Here we take a complementary approach and look for the presence of molecular outflows in order to separate the protostellar cores from the starless population. As outflow activity is thought to begin almost as soon as the formation of a hydrostatic core, outflows are a good indicator of the presence of embedded protostars.

* Figs. 9 and 10 are only available in electronic form at http: //www . aanda.org

$\star \star$ Datacube data in FITS are only available in electronic form at the CDS via anonymous ftp to

cdsarc.u-strasbg.fr $(130.79 .128 .5)$ or via

http://cdsweb.u-strasbg.fr/cgi-bin/qcat?J/A+A/472/187
In addition, studying molecular outflows is an important way to get information on the accretion/mass ejection process which drives protostellar evolution. Several important studies have shown that the outflow force or momentum flux $F_{\mathrm{CO}}$ is correlated with both mass and luminosity of the protostellar core (Cabrit \& Bertout 1992; Bontemps et al. 1996), a correlation which recently has been shown to hold over several orders of magnitude (Churchwell 1997; Beuther et al. 2002; Zhang et al. 2005) and held to be strong evidence for a common protostellar formation mechanism for high- and low-mass sources alike. The details of mass accretion and ejection, however, remain uncertain with competing mechanisms for the interaction between mass flow and magnetic fields in the inner parts of accretion disks (Shu et al. 1994; Wardle \& Koenigl 1993; Pelletier \& Pudritz 1992) and considerable uncertainty on how the accretion rate varies with time. One certainty, however, seems to be that the accretion rate and the momentum output in the jet/wind are strongly linked (Shu et al. 1994; Ferreira \& Pelletier 1995; Bontemps et al. 1996), and therefore, assuming a roughly constant mass entrainment fraction, one can use the outflow momentum as a surrogate measure of the momentum in the jet/wind, and therefore the mass accretion rate onto the protostar.

We therefore set out to make small outflow maps around the submm cores in Perseus to determine their outflow properties. 
With a consistent dataset for a moderately large sample drawn from one molecular cloud, we can look statistically at the outflow detection rate and the variation of the momentum flux with other parameters.

We assume a distance of $320 \mathrm{pc}$ for the Perseus molecular cloud based on the Hipparcos distance of IC 348 (de Zeeuw et al. 1999), consistent with our earlier paper (Paper I), though readers should be aware that other recent studies (Kirk et al. 2006; Enoch et al. 2006) have assumed a closer distance of $250 \mathrm{pc}$ based on extinction studies (Černis \& Straižys 2003, and references therein).

In Sect. 2 we describe the observations. Section 3 contains the results and an examination of the detection statistics. Momentum fluxes and outflow evolution are considered in Sect. 4. In Sect. 5 we discuss the derived outflow mass loss rates and the implications for core destruction timescales given the core masses implied by the submm emission (Paper I; Paper II). Finally, our conclusions are given in Sect. 6.

\section{2. ${ }^{12} \mathrm{CO}$ 3-2 outflow observations}

Outflow maps were made in ${ }^{12} \mathrm{COJ}=3-2$ in the years 2000-2003 using RxB3 on the James Clerk Maxwell Telescope (JCMT). ${ }^{12} \mathrm{CO} \mathrm{3-2}$ is a better tracer of molecular outflows than lower-J transitions as it is more sensitive to warm outflow than cold ambient emission. The sample consisted of contiguous areas around the known clusters B1, IC 348, L1448, L1455, NGC 1333 (Knee \& Sandell 2000), and a number of isolated submm cores. The map areas were extended $2^{\prime} \times 2^{\prime}$ around each isolated source or tiled to cover the cluster regions. The submm cores which lie within the outflow maps are listed in Table 1. Positions and further information on these cores can be found in Paper I and Paper II. The beamsize at $345 \mathrm{GHz}$ is $14^{\prime \prime}$ and the maps were fully sampled at $5^{\prime \prime}$ spacing in RA and Dec. A correction for main beam efficiency of $\eta_{\mathrm{MB}}=0.63$ was applied. The noise level was $0.5 \mathrm{~K}$ or less on $625 \mathrm{kHz}\left(0.54 \mathrm{~km} \mathrm{~s}^{-1}\right)$ channels. The weather conditions were various and resulting system temperatures were $350-500 \mathrm{~K}$.

The ${ }^{12} \mathrm{CO} 3-2$ data for NGC 1333 were published in Knee \& Sandell (2000), and kindly made available by the authors. The spatial sampling of the NGC 1333 observations was $10^{\prime \prime}$ rather than the $5^{\prime \prime}$ of the more recent observations. In order to match the other datasets as closely as possible for analysis, the maps were resampled onto a $5^{\prime \prime}$ grid. This was done by interpolating with a $14^{\prime \prime}$ Gaussian truncated at $40^{\prime \prime}$. This results in a map with the same intensity $\left(T_{\mathrm{MB}}\right)$ scale but with $20^{\prime \prime}$ resolution.

The sample (Table 1) was selected before the analysis of the corresponding SCUBA data was complete, and therefore is biased towards the obvious bright clusters and previously known protostars. In Fig. 2 we show the outflow sample (marked by stars) on a plot of luminosity vs. core mass for the entire population of submm cores in Perseus (Paper II). The sample is indeed biased towards high luminosities with no outflow maps of sources below $0.25 L_{\odot}$. The proportion of the total number of cores decreases with decreasing mass. However, the fraction of $L_{\text {bol }}>0.25 L_{\odot}$ cores studied remains fairly constant except for the very lowest mass sources below our completeness limit at $10 \mathrm{~K}$ of $M_{\mathrm{env}}<0.5 M_{\odot}(5 \sigma)$. For the very highest mass sources, $M_{\text {env }}>12 M_{\odot}$, all sources were observed as there were no lowluminosity sources in the SCUBA sample. Thus the most significant bias of the sample is its lack of low luminosity sources.

Small outflow maps provide a good estimate of the momentum flux (Fuller \& Ladd 2002; Mangum et al. 1998) but, except for very small flows, do not provide estimates of the full extent, mass, kinetic energy, or dynamical timescale of outflows. Our map radius of $1^{\prime}$ corresponds to $0.1 \mathrm{pc}$ assuming a distance of $320 \mathrm{pc}$ for the Perseus molecular clouds, or a dynamical time of 2000 years for an outflow advancing at $50 \mathrm{~km} \mathrm{~s}^{-1}$.

\section{Outflow activity}

Maps of the outflows superimposed on the submm continuum map are shown in Fig. 1. We identified sources with outflows based on the objective criterion of ${ }^{12} \mathrm{CO}$ linewings more than $1.5 \mathrm{~K}(3 \sigma)$ at $3 \mathrm{~km} \mathrm{~s}^{-1}$ from the line centre (as measured in $\mathrm{C}^{18} \mathrm{O}$ ). Of the 51 sources in Table 1 observed in ${ }^{12} \mathrm{CO} 3-2$, 37 sources $(73 \%)$ were classified as outflows on this basis. Spectra for these sources at the source position and the outflow lobes are shown in Fig. A.1 (online version only). In some cases the outflow lobe spectra are missing due to confusion or because the driving source lies at the edge of the mapped area. From the maps (Fig. 1), we identify 13 cases where the broad linewings may be wholly or partly due to nearby flows driven by other sources in crowded regions. These uncertain flows are marked with "y?" in Table 1 . The remaining 14 sources show no evidence for outflow activity in the form of either line wings or red/blueshifted lobes, as shown in Fig. A.2 (online version only).

All but four of the sources with outflows on the basis of linewings also have a near-infrared detection from Spitzer IRAC (Paper II), (Jørgensen et al. 2007). The exceptions are 17, 40, 51 (SK16) and 53 (SK26), all of which are classified as starless (Table 1). In all of these cases the linewings are likely due to confusion. In the following analysis of the outflow properties, we leave out these four sources, assuming that they are indeed starless. For most of the protostars it is the driving source which is being detected by Spitzer but in some cases the infrared emission may be due to HH objects (examples are HH211 (12), and (15)). In 9 of the 13 uncertain outflow sources, their protostellar nature is confirmed by IR detections. All but one of the sources with Spitzer IRAC detections and observed in $\mathrm{CO}$ have molecular outflows. The exception is in NGC 1333: the Class 0 source 61 lies to the north of the luminous Herbig Ae-Be star NGC 1333 IRAS 8/SSV3 and B star IRAS 9/BD+30 549 and the interaction with radiation and winds from these sources may well explain the outflow non-detection. The detection of outflows from all but one Spitzer-confirmed protostar shows that both types of observation are similarly effective in identifying protostars among submm cores. Outflow-based identification avoids the Spitzer confusion with extragalactic objects but can be difficult in crowded regions where the outflows overlap.

\subsection{Outflow detection statistics}

If we consider the outflows as a function of core mass (Fig. 3, top), we find that the fraction of sources with outflows decreases with decreasing core mass. In order to estimate our ability to detect outflows in the lower mass bins, we performed the following simulation. We considered the 14 outflow sources in the three highest mass bins $\left(M_{\mathrm{env}}>1 M_{\odot}\right)$. We then simulated outflows from lower mass cores by scaling the outflow spectra (Fig. A.1, online version only) for these 15 sources according to the modelled core mass as (intensity/original intensity) = (model mass/original mass) ${ }^{1.5}$. The motivation for this power law scaling comes from Fig. 4 which shows a rough correlation between momentum flux and core mass with a gradient of $1.5 \pm 0.5$ (see Sect. 4). We also formed the simulated outflow maps by multiplying the original $(x, y, v)$ datacube by these scaling factors and adding back on the unscaled noise levels (from 

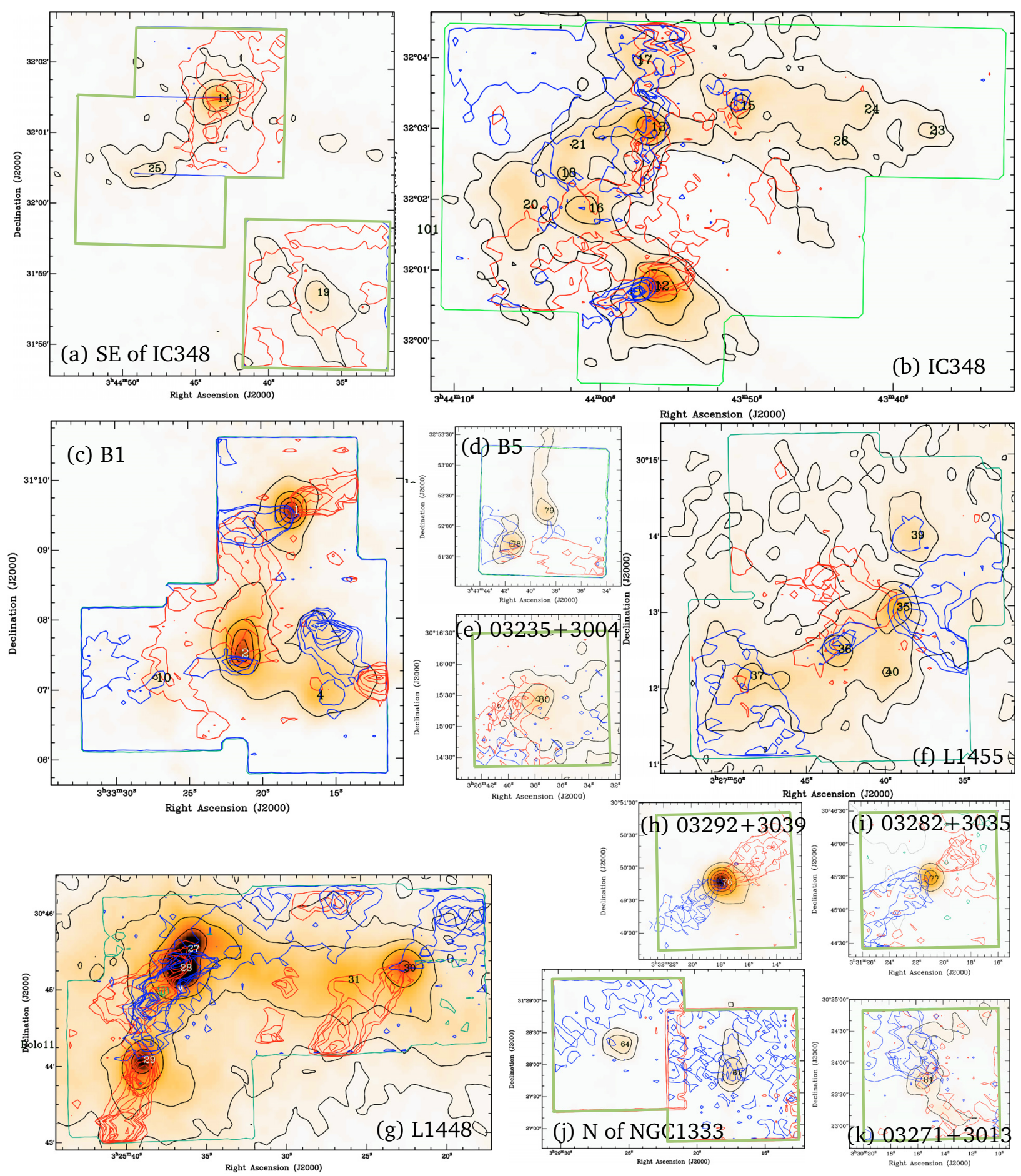

Fig. 1. ${ }^{12}$ CO 3-2 outflow maps. Colourscale: $850 \mu \mathrm{m}$ SCUBA map with black contours at 100, 200, 400, 800, $1600 \mathrm{mJy} / \mathrm{beam}$. The area observed in ${ }^{12} \mathrm{CO} 3-2$ is outlined in green. Outflows are displayed as a renzogram, equivalent to superimposing a series of channel maps each with one contour at a fixed intensity level (e.g. Kregel et al. 2004). a) Region to the SE of IC 348 The renzogram intensity level is $3 \mathrm{~K} \mathrm{~km} \mathrm{~s}^{-1}$ and included channels from $-5-7 \mathrm{~km} \mathrm{~s}^{-1}$ (blue) and 11-15 $\mathrm{km} \mathrm{s}^{-1}$ (red). b) IC 348 including HH211 (12) and IC $348 \mathrm{mms}$ (13): $2 \mathrm{~K} \mathrm{~km} \mathrm{~s}^{-1}$ from -5-6 km s (blue) and 11-15 km s${ }^{-1}$ (red). c) B1 including B1-c (1), B1-bN/S (2), b1-d (4) and IRAS 03301+3057 (7): $3 \mathrm{~K} \mathrm{~km} \mathrm{~s}^{-1}$, from -5-4 km s${ }^{-1}$ (blue) and $9-15 \mathrm{~km} \mathrm{~s}^{-1}$ (red). d) B5 IRS1 (78) and (79) $2.5 \mathrm{~K} \mathrm{~km} \mathrm{~s}^{-1},-5-8$ (blue) and 12-15 km s (red). e) IRAS 03235+3004 (80) $2.0 \mathrm{~K} \mathrm{~km} \mathrm{~s}^{-1}$, $-5-3$ (blue) and 6-15 $\mathrm{km} \mathrm{s}^{-1}$ (red). f) L1455 including RNO15 (35), PP9 (37), and L1455 FIR2 (39): $1.8 \mathrm{~K} \mathrm{~km} \mathrm{~s}^{-1}$ from -5-2 km s (blue) $^{-1}$ and 11-18 $\mathrm{km} \mathrm{s}^{-1}$ (red). g) L1448 including L1448 N/NW (27), L1448 C (28) and L1448 IRS2 (30): $3.0 \mathrm{~K} \mathrm{~km} \mathrm{~s}^{-1}$ from -5-0 km s${ }^{-1}$ (blue) and $8-15 \mathrm{~km} \mathrm{~s}^{-1}$ (red). h) IRAS $03292+3039$ (76): $2.0 \mathrm{~K} \mathrm{~km} \mathrm{~s}^{-1},-5-4$ (blue) and 9-15 km s (red). i) IRAS 03282+3035 (77): $3.0 \mathrm{~K} \mathrm{~km} \mathrm{~s}$ - $-5-5$ (blue) and $7-15 \mathrm{~km} \mathrm{~s}^{-1}$ (red). j) Region in the $\mathbf{N}$ of NGC 1333. $2 \mathrm{~K} \mathrm{~km} \mathrm{~s}^{-1}$ from $-5-1 \mathrm{~km} \mathrm{~s}^{-1}$ (blue) and $12-15 \mathrm{~km} \mathrm{~s}^{-1}$ (red). k) $\mathbf{0 3 2 7 1 + 3 0 1 3}(81)$ $1.0 \mathrm{~K} \mathrm{~km} \mathrm{~s}^{-1},-5-6$ (blue) and $8-15 \mathrm{~km} \mathrm{~s}^{-1}$ (red) $)^{2}$.

sampling the noise in the outer velocity channels of the unscaled maps). Several scaling factors were used, corresponding

${ }^{2}$ The datacube data in FITS are available at CDS. to moving the core masses an integer number of mass bins to the left. By this means we generated an artificial population of spectra and maps of outflows for cores in the range 0.6-50 $M_{\odot}$. 


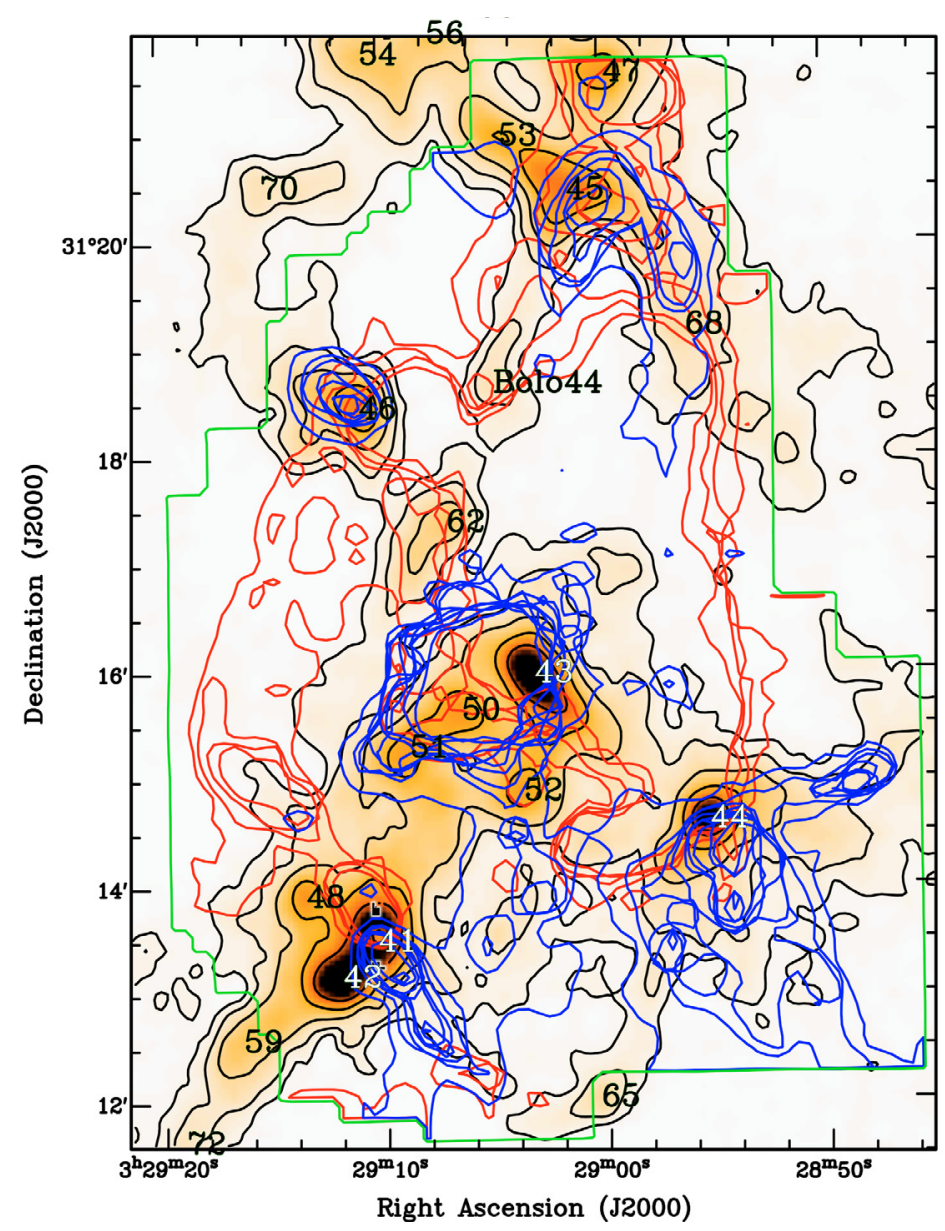

Fig. 1. continued. NGC 1333 from data first published in Knee \& Sandell (2000). The renzogram intensity level is $3 \mathrm{~K} \mathrm{~km} \mathrm{~s}^{-1}$ and included channels from $-5-3 \mathrm{~km} \mathrm{~s}^{-1}$ (blue) and $12-18 \mathrm{~km} \mathrm{~s}^{-1}$ (red). $850 \mu \mathrm{m}$ contours and colourscale are as before.

We tested the spectra according to our criterion for linewings $\left(>1.5 \mathrm{~K}\right.$ at $3 \mathrm{~km} \mathrm{~s}^{-1}$ from line centre). We also additionally displayed these maps using our usual visualisation software kvis (Gooch 1996) and considered whether we could visually detect the resulting outflows above the noise. In all cases where the linewings satisfied the objective criteria, we could see the outflows in the maps. The detection statistics for our simulated population are given in Table 2.

There is a decline in our detection ability at lower mass, and in the lowest mass bins we detect few of the model outflows. In the lowest two mass ranges in Fig. $3,0.4-2.5 M_{\odot}, 0$ and 1 respectively out of 14 of the simulated low-mass outflows were detected. In the next highest mass bin $\left(2.5-6 M_{\odot}\right)$, the detection rate improved and $29 \%$ of simulated outflows were detected. The mean detection rate below $6 M_{\odot}$ is predicted to be $12 \%$, or roughly 1 in 8 outflows detected.

The outflow detection rate predicted by the simulation is lower than the actual detection rate for all sources below $6 M_{\odot}$. This can be seen in the lowest three mass bins of Table 2 . This is true even if we include all the submm cores from the SCUBA survey, and not just those with existing outflow observations. We have already detected 14 outflows among 75 submm cores with masses below $6 M_{\odot}$ (Fig. 2), or $19 \%$ compared to an average of $12 \%$ for the simulated flows. This suggests that our assumptions about how outflow linewings scale with core mass are pessimistic, and we are more likely to detect flows than the simulation predicts - due to higher optical depth in more massive flows, for example.
The bottom panel of Fig. 3 shows our best estimate of the true fraction of outflow sources in each mass bin, corrected for the detection statistics using (true outflow fraction) $=$ (fraction of outflows detected)/(detection probability in this mass bin). The calculation of the uncertainties (greyscales) shown in the figure is given in Appendix A. In mass bins where the observed detection rate equals or exceeds the simulated rate, this model would predict that all the apparently starless cores contain undetected protostars. Only in the $6-16 M_{\odot}$ mass range is the predicted number of outflow sources smaller than the number of apparently starless cores. If this model is correct, then we fail to detect outflows from the majority of protostars at masses $<6 M_{\odot}$.

Thus, the modelling suggests that the low number of outflow detections from low mass cores is a selection effect.

If this is the case then this outflow survey and Spitzer c2d Evans et al. (2003) must have very similar sensitivities to embedded protostars as only one mid-IR detected sources in the sample had no corresponding outflow and conversely only four outflow sources had no mid-IR detection. Therefore any selection effect must operate both in the infrared and molecular lines. Orientation could help produce such close agreement as infrared sources are hard to detect where disks are edge-on and outflows are hard to detect when they lie close to the plane of the sky. Assuming random outflow orientation, though, the number of infrared non-detections due to edge-on disks (within $10^{\circ}$ of the line-of-sight) can only be $\sim 20 \%$ of the total protostellar fraction.

This modelling suggests that the low-mass cores without outflow or Spitzer detections may also be protostellar, but of course 
Table 1. Sources with outflow observations and resulting momentum fluxes. For each source we list the core number and name (see Hatchell et al. 2007, for positions and core properties), the outflow status; the core ambient velocity $v_{\mathrm{LSR}}$, derived from $\mathrm{C}^{18} \mathrm{O}$, the maximum radius used in the momentum flux calculation, $\theta_{2}\left(\theta_{1}\right.$ was set to zero); and the resulting momentum flux and uncertainty. Of the 51 outflow sources, 24 have definite outflows, 14 have no outflow detection, and 13 (9 with infrared detections) have possible but confused outflows.

\begin{tabular}{|c|c|c|c|c|c|c|}
\hline $\mathrm{Nr}$. & Name $^{1}$ & $\begin{array}{l}\text { Outflow } \\
(\mathrm{y} / \mathrm{n} ?)\end{array}$ & Class $^{2}$ & $\begin{array}{l}v_{\mathrm{LSR}} \\
\mathrm{km} \mathrm{s}^{-1}\end{array}$ & $\begin{array}{l}\theta_{2} \\
\prime \prime\end{array}$ & $\begin{array}{r}F_{\mathrm{CO}} \\
10^{-7} M_{\odot} \mathrm{km} \mathrm{s}^{-1} \mathrm{yr}^{-1}\end{array}$ \\
\hline 1 & b1-c & $\mathrm{y}$ & 0 & 6.4 & 12.0 & $1.7(0.8)$ \\
\hline 2 & b1-bS & $\mathrm{y}$ & 0 & 6.8 & 30.0 & $12.3(1.0)$ \\
\hline 4 & b1-d & $\mathrm{y}$ & 0 & 6.7 & 15.0 & $1.8(0.3)$ \\
\hline 7 & IRAS $03301+3057$ & $\mathrm{y}$ & I & 6.6 & 30.0 & $12.6(0.8)$ \\
\hline 10 & B1 SMM11 & $\mathrm{y}$ & I & 6.6 & 30.0 & $4.4(0.4)$ \\
\hline 12 & HH211 & $\mathrm{y}$ & 0 & 8.8 & 30.0 & $6.2(0.8)$ \\
\hline 13 & IC 348 MMS & $\mathrm{y}$ & 0 & 8.6 & 30.0 & $5.1(0.5)$ \\
\hline 14 & & $\mathrm{y}$ ? & I & 9.1 & 10.0 & $1.2(0.3)$ \\
\hline 15 & IC 348 SMM3 & $\mathrm{y}$ & 0 & 8.8 & 30.0 & $0.6(0.2)$ \\
\hline 16 & & $\mathrm{n}$ & $S$ & 8.8 & - & -- \\
\hline 17 & & $\mathrm{y} ? \mathrm{c} 13^{3}$ & $S$ & 8.4 & 10.0 & $0.8(0.6)$ \\
\hline 18 & & $\mathrm{n}$ & $\mathrm{S}$ & 8.6 & - & -- \\
\hline 19 & & $\mathrm{n}$ & $\mathrm{S}$ & 9.5 & - & -- \\
\hline 20 & & $\mathrm{n}$ & $\mathrm{S}$ & 8.5 & - & -- \\
\hline 21 & & $\mathrm{n}$ & $\mathrm{S}$ & 8.6 & - & -- \\
\hline 23 & & $\mathrm{n}$ & $\mathrm{S}$ & 9.0 & - & -- \\
\hline 24 & & $\mathrm{n}$ & $\mathrm{S}$ & 8.7 & - & -- \\
\hline 25 & & $\mathrm{n}$ & $\mathrm{S}$ & 9.2 & - & -- \\
\hline 26 & & $\mathrm{n}$ & $\mathrm{S}$ & 8.8 & - & -- \\
\hline 27 & L1448 NW & $\mathrm{y}$ & 0 & 4.4 & 30.0 & $27.5(3.0)$ \\
\hline 28 & L1448 N A/B & $\mathrm{y}$ & 0 & 4.4 & 30.0 & $36.4(2.9)$ \\
\hline 29 & L1448 C & $\mathrm{y}$ & 0 & 4.8 & 30.0 & $20.9(1.5)$ \\
\hline 30 & L1448 IRS2 & $\mathrm{y}$ & 0 & 4.1 & 30.0 & $10.1(0.8)$ \\
\hline 31 & & $y ? c 30^{3}$ & 0 & 4.1 & 15.0 & $3.6(0.5)$ \\
\hline 35 & L1455 FIR4 & $\mathrm{y}$ & I & 5.3 & 30.0 & $9.4(0.6)$ \\
\hline 36 & & $\mathrm{y}$ & 0 & 5.8 & 20.0 & $6.6(0.5)$ \\
\hline 37 & L1455 PP9 & $\mathrm{y}$ ? & I & 5.3 & 30.0 & $3.2(0.4)$ \\
\hline 39 & L1455 FIR1/2 & $\mathrm{y}$ ? & I & 5.3 & 20.0 & $1.8(0.2)$ \\
\hline 40 & & y?c $35,36^{3}$ & $\mathrm{~S}$ & 5.4 & 30.0 & $2.6(0.3)$ \\
\hline 41 & NGC 1333 IRAS 4A & $\mathrm{y}$ & 0 & 7.6 & 30.0 & $45.5(2.9)$ \\
\hline 42 & NGC 1333 IRAS 4B & $\mathrm{y}$ & 0 & 7.5 & 10.0 & $10.4(1.6)$ \\
\hline 43 & NGC 1333 SVS13 & $\mathrm{y}$ & I & 7.9 & 30.0 & $195.9(14.2)$ \\
\hline 44 & NGC 1333 IRAS 2A & $\mathrm{y}$ & 0 & 7.7 & 30.0 & $72.7(4.4)$ \\
\hline 45 & NGC 1333 SK24 & $\mathrm{y}$ & I & 7.6 & 30.0 & $50.3(3.4)$ \\
\hline 46 & NGC 1333 SK20/21 & $\mathrm{y}$ & 0 & 7.9 & 30.0 & $59.5(3.3)$ \\
\hline 47 & NGC 1333 SK31 & $\mathrm{y}$ & 0 & 7.6 & 30.0 & $19.2(1.9)$ \\
\hline 48 & NGC 1333 IRAS 4C & $y ? c 41,42^{3}$ & 0 & 7.6 & 20.0 & $2.7(1.6)$ \\
\hline 50 & NGC 1333 SK15 & $\mathrm{y} ? \mathrm{c} 43,51^{3}$ & I & 7.9 & 20.0 & $166.2(10.9)$ \\
\hline 51 & NGC 1333 SK16 & $y ? c 43,50^{3}$ & $\mathrm{~S}$ & 7.6 & 20.0 & $54.5(8.2)$ \\
\hline 52 & NGC 1333 SK14 & $\mathrm{y} ? \mathrm{c} 43,50^{3}$ & 0 & 7.6 & 30.0 & $32.5(2.6)$ \\
\hline 53 & NGC 1333 SK26 & $\mathrm{y} ? \mathrm{c} 45^{3,4}$ & S & 7.6 & 20.0 & $4.4(0.6)$ \\
\hline 61 & & $\mathrm{n}$ & 0 & 7.6 & - & -- \\
\hline 62 & NGC 1333 SK18 & $y ? c 46^{3}$ & 0 & 7.9 & 20.0 & $25.6(4.1)$ \\
\hline 64 & NGC 1333 Per 4A3/4D & $\mathrm{n}$ & $\mathrm{S}$ & 7.4 & - & -- \\
\hline 68 & NGC 1333 MBO146 & $\mathrm{y} ? \mathrm{c} 45^{3}$ & 0 & 7.7 & 30.0 & $27.5(2.3)$ \\
\hline 76 & IRAS $03292+3039$ & $\mathrm{y}$ & 0 & 6.9 & 10.0 & $1.2(0.5)$ \\
\hline 77 & IRAS $03282+3035$ & $\mathrm{y}$ & 0 & 7.2 & 30.0 & $4.8(0.5)$ \\
\hline 78 & B5 IRS1 & $\mathrm{y}$ & I & 10.0 & 10.0 & $1.5(0.2)$ \\
\hline 79 & & $\mathrm{n}$ & $\mathrm{S}$ & 10.0 & - & -- \\
\hline 80 & IRAS $03235+3004$ & $\mathrm{n}$ & I & 5.0 & - & -- \\
\hline 81 & B1 SMM1 & $\mathrm{n}$ & I & 5.9 & - & -- \\
\hline
\end{tabular}

${ }^{1}$ Common name. For positions, further names, references and further data see Paper II.

${ }^{2}$ From SED-based evolutionary indicators (Paper II).

${ }^{3}$ Sources of potential confusion.

${ }^{4}$ At edge of mapped area. Possible S blue lobe.

does not prove that they are. For one thing, the assumptions in our model about how outflow masses vary with core mass may not be correct. Only further searching for outflows, infrared or radio sources towards the apparently starless cores can determine the true nature of the apparently starless cores. Note that such observations would need to be with higher sensitivity than this outflow survey, the Spitzer Cores to Disks programme (Evans et al. 2003), or its successor, the Spitzer Gould's Belt survey, in order to identify less luminous protostars. 
$L_{\text {bol }}$ vs. $M_{\text {env }}$

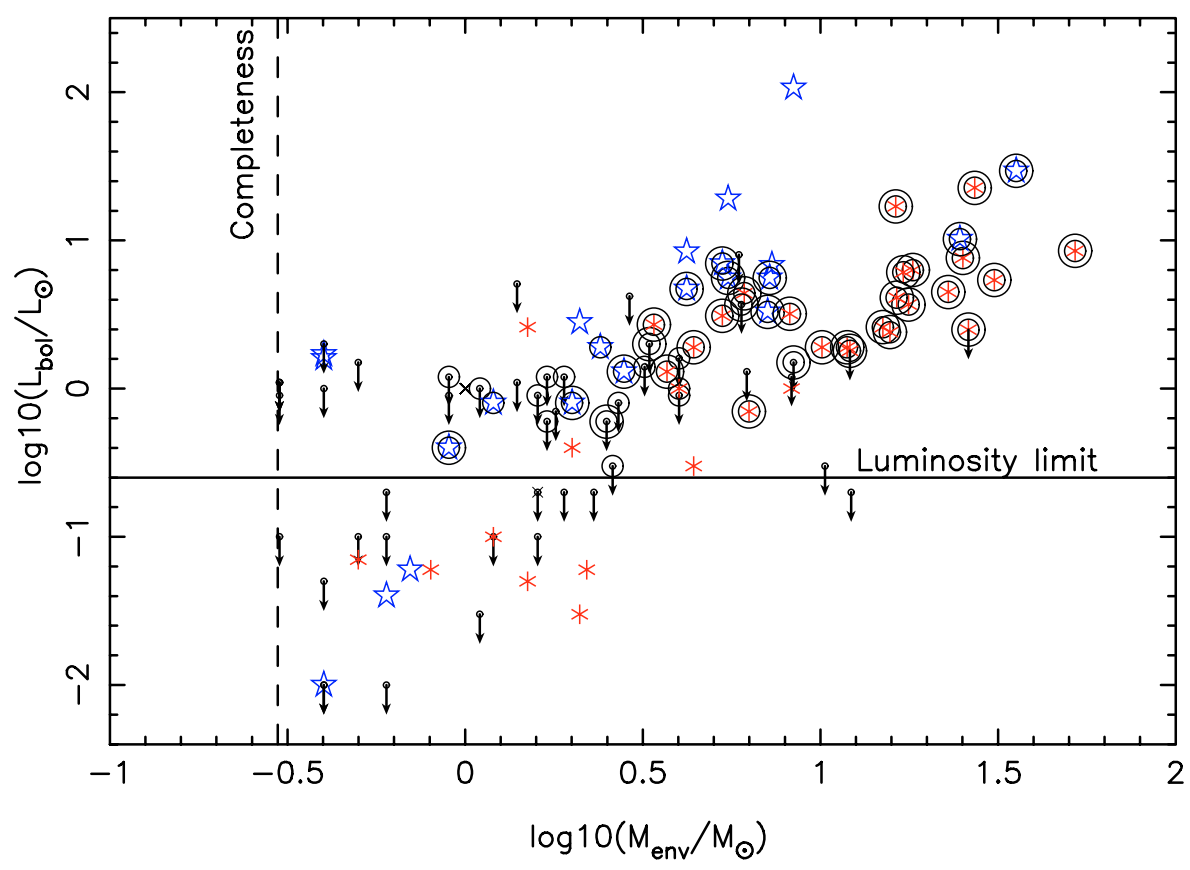

Fig. 2. $L_{\mathrm{bol}}$ vs. $M_{\mathrm{env}}$ for all submm cores in Perseus (Paper I; Paper II) with ${ }^{12} \mathrm{CO}$ targets marked by small black circles and outflow detections additionally marked by large black circles. Class 0 sources are marked as asterisks $(*$, red) and Class I by open stars (blue). The remaining sources (dots) are assumed starless. The horizontal line at $0.25 L_{\odot}$ marks the lower luminosity limit for the outflow sample, and the vertical dashed line at $0.3 M_{\odot}$ marks the $3 \sigma$ detection limit for the SCUBA survey.
Table 2. Fraction of simulated outflows detected in each mass bin, compared to observed detection fraction.

\begin{tabular}{l|llllll}
\hline \hline $\log _{10}\left(M / M_{\odot}\right)$ & -0.2 & 0.2 & 0.6 & 1.0 & 1.4 & 1.8 \\
\hline Simulated maps & 14 & 14 & 14 & 14 & 11 & 1 \\
Sim. detections & 0 & 1 & 4 & 13 & 11 & 1 \\
Sim. rate & $0 \%$ & $7 \%$ & $29 \%$ & $93 \%$ & $100 \%$ & $100 \%$ \\
Obs. rate & $50 \%$ & $18 \%$ & $50 \%$ & $64 \%$ & $100 \%$ & $100 \%$ \\
\hline
\end{tabular}

\section{Momentum fluxes}

Outflow momentum fluxes (or outflow luminosities) are given in Table 1 for all the possible outflow sources. The method is developed from the momentum flux per beam calculation of Fuller \& Ladd (2002), rather than calculating the momentum flux within an annulus (Bontemps et al. 1996), though the two methods should yield comparable results. The momentum flux crossing a cylinder radius $\theta_{r}$ perpendicular to the plane of the sky and centred on the source, contributed by the mass within one beam, is calculated individually for each beam as:

$\dot{P}=\sum_{v_{\mathrm{obs}}} \frac{M_{\mathrm{B}}\left(v_{\mathrm{obs}}\right) v_{\mathrm{obs}} / \cos i}{D \theta_{\mathrm{B}} / v_{\mathrm{obs}} \tan i}$

where $v_{\mathrm{obs}}=\left|v_{i}-v_{\mathrm{LSR}}\right|$ is the observed velocity offset from the core systemic velocity $v_{\text {LSR }}$ (determined from $\mathrm{C}^{18} \mathrm{O}$ observations of the same region at $1^{\prime}$ resolution (Paper I)), $i$ is the angle the outflow makes with the line-of-sight, $D$ the source distance and $\theta_{\mathrm{B}}$ the beam FWHM. The numerator gives the momentum in the beam $\left(v=v_{\text {obs }} / \cos i\right.$ is the outflow radial velocity) and the denominator the time taken to cross the beamwidth at velocity $v$. The sum is over velocity channels.

The mass within one beam $M_{\mathrm{B}}$ is calculated from the $\mathrm{CO}$ integrated intensity over the velocity channel $I_{\mathrm{CO}} \Delta v$ assuming a $\mathrm{CO}$ abundance of $X_{\mathrm{CO}}=10^{-4}$ (Frerking et al. 1982; Wilson \& Rood 1994) and a LTE excitation at an outflow temperature of $50 \mathrm{~K}$ resulting in $N_{\mathrm{H}_{2}}=2.5 \times 10^{19}\left(\int T_{\mathrm{MB}}^{*} \mathrm{~d} v / \mathrm{K} \mathrm{km} \mathrm{s}^{-1}\right) \mathrm{cm}^{-2}$.
A temperature of $50 \mathrm{~K}$ is chosen as there is increasing evidence that outflows contain warm gas (Hatchell et al. 1999b,a; Nisini et al. 2000; Giannini et al. 2001) but the conversion is relatively insensitive to the temperature, increasing by only $40 \%$ over the temperature range $20-100 \mathrm{~K}$. The mass within one beam is calculated as $M_{\mathrm{B}}=(\pi / 4) D^{2} \theta_{\mathrm{B}}^{2} N_{\mathrm{H}_{2}} m_{\mathrm{H}_{2}}$.

The momentum flux contributions from individual beams are then summed in annuli, according to:

$$
\begin{aligned}
F_{\mathrm{CO}} & =\sum_{\text {annulus }} \frac{2 \pi \theta_{r}}{N_{\mathrm{pix}} \theta_{B}} \dot{P} \\
& =\sum_{\theta_{1}<\theta_{r}<\theta_{2}} \frac{\pi^{2} \theta_{r} D}{2 N_{\mathrm{pix}}} \sum_{v_{\mathrm{obs}}} N_{\mathrm{H}_{2}}\left(v_{\mathrm{obs}}\right) m_{\mathrm{H}_{2}} v_{\mathrm{obs}}^{2} \frac{\sin i}{\cos ^{2} i}
\end{aligned}
$$

where $\theta_{r}$ is the angular distance of each pixel from the outflow centre, $D$ is the source distance, and the average is over pixels between radii $\theta_{1}$ and $\theta_{2}$ with $N_{\text {pix }}$ the number of pixels between those radii. As the contribution to the momentum flux is calculated individually for each beam, and varies little between neighbouring annuli, we set the inner radius to zero. The factor $2 \pi \theta_{r} / N \theta_{B}$ corrects for the overcounting because the $14^{\prime \prime}$ beam size is larger than the $5^{\prime \prime}$ pixel size.

The driving source positions were taken to be the SCUBA emission peaks from Paper I. To reduce the contribution due to emission outside the outflow region, the outflow areas to be included in the momentum flux calculation were selected by masking with ${ }^{12} \mathrm{CO}$ integrated intensity at a cutoff of 3 times the noise level in the integrated intensity map. We default to calculating the momentum flux within a circle of outer radius $30^{\prime \prime}$ unless it was clear from the map that the outflow was shorter than this or there was potential confusion within this radius, in which case the radius of the circle was reduced. In practice we find the choice of circle or annulus and the radius of the circle (except for the case of confusing flows) makes little difference to the resulting momentum fluxes, as expected at radii where there is no large variation in the injection of momentum. 

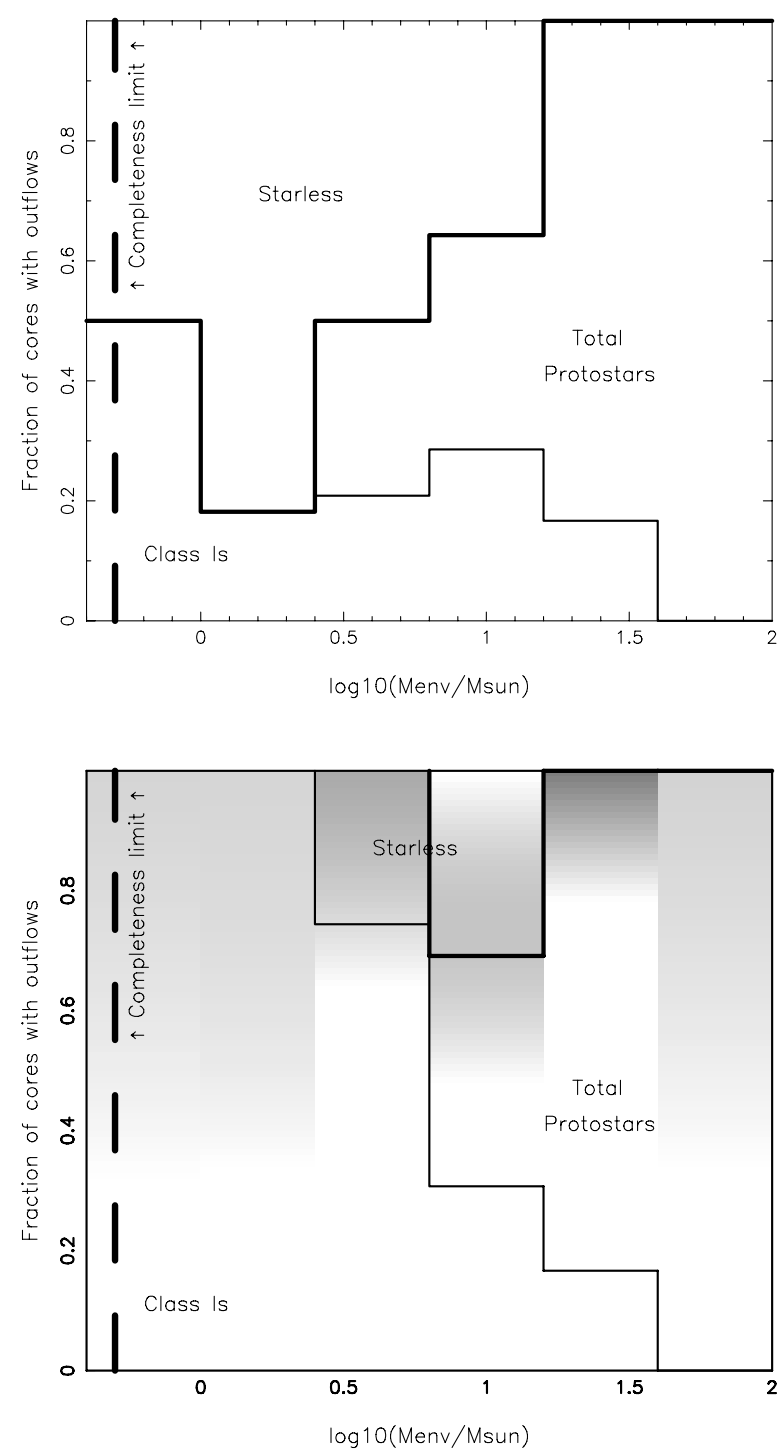

Fig. 3. Top: fraction of outflow sources as a function of core mass for sources with definite outflows (solid thick line). The thin line shows the number of Class I sources as classified in Paper II. The ratio of protostars to starless cores decreases with decreasing mass. Bottom: as above with the fraction of protostars corrected for the outflow detection statistics in that mass bin. The greyscale gives the probability distribution of the fraction of protostars (see Appendix).

The inner velocity channels, contaminated by ambient emission, were cut out to $\pm 2 \mathrm{~km} \mathrm{~s}^{-1}$ and the highest velocity considered was $\pm 15 \mathrm{~km} \mathrm{~s}^{-1}$. Ideally, for a fair comparison of the momentum flux between strong (massive) and weak flows, one wants good signal-to-noise $(\mathrm{S} / \mathrm{N})$ in the linewings out to similar velocities. With roughly constant sensitivity and line strengths varying by an order of magnitude the strong linewings can be measured with good $\mathrm{S} / \mathrm{N}$ to higher velocities in the stronger flows. Adjusting the velocity range on a flow-by-flow basis according to where the $\mathrm{S} / \mathrm{N}$ is good therefore introduces a strong bias in momentum flux towards massive flows. Not only are these more massive but also more of the high velocity channels are included in the calculation. Therefore we consider it fairer to use the same velocity cut for each source, even though this excludes the contribution from the highest velocity channels in all flows.
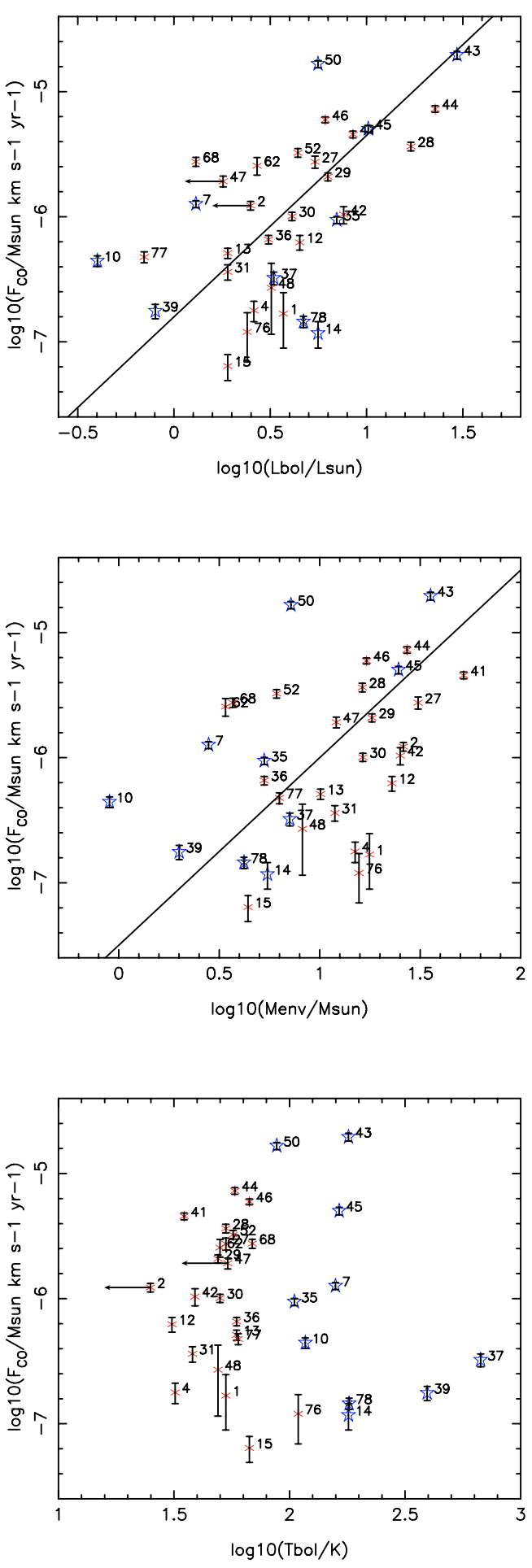

Fig. 4. Relationships between momentum flux and (top to bottom) bolometric luminosity $L_{\mathrm{bol}}$, core mass $M_{\mathrm{env}}$, and bolometric temperature $T_{\text {bol }}$. Sources marked by open stars (blue) and asterisks (red) are Class I and Class 0 respectively based on the SED (Paper II). The top two panels show best fits corresponding to $F_{\mathrm{CO}} \propto L_{\mathrm{bol}}{ }^{1.5}$ and $F_{\mathrm{CO}} \propto M_{\mathrm{env}}{ }^{1.5}$ respectively. Uncertainties on $F_{\mathrm{CO}}$ (see Sect. 4) are shown; uncertainties on $L_{\text {bol }}$ are typically $30 \%, M_{\text {env }}$ at least a factor 2 , and $T_{\text {bol }}$ a few Kelvin.

The uncertainties on the momentum flux, listed in Table 1, are the root sum-of-squares of the uncertainties of each contributing pixel. The pixel uncertainty is taken to be the difference 
from the mean momentum flux in the contributing (integrated intensity $>3 \sigma$ ) region.

We have not applied a correction factor for either inclination or optical depth, unlike Bontemps et al. (1996). Therefore our momentum fluxes are strictly lower limits. Bontemps et al. (1996) point out that inclination $i$ reduces the momentum flux by a factor $\sin i / \cos ^{2} i=2.9$ for a mean angle of 57.3 degrees. For an optical depth $\tau$, uncorrected CO column densities are underestimated by a factor $\tau /(1-\exp (-\tau))$ or a typical factor of 3.5 so that true momentum fluxes are typically a factor of 10 greater than measured. Outflow optical depths are high even in the $J=3-2$ line with with $\tau_{3-2}>4$ in the line wings (Margulis \& Lada 1985; Hogerheijde et al. 1998).

The (uncorrected) momentum flux results for all the possible outflow sources are given in Table 1. Comparing the sources in common, our momentum fluxes are typically lower by a factor of a few than previous analyses by Bontemps et al. (1996) (taking the optical depth/inclination correction into account) and Knee $\&$ Sandell (2000). The main reason for this is that we assume the outflows are warm $(50 \mathrm{~K})$, which reduces mass estimates by a factor of $\sim 4$ over the $10 \mathrm{~K}$ assumed by Bontemps et al. (1996). This is a systematic difference, reducing the momentum fluxes for all sources by the same fraction. For flows with high velocity gas, we can also underestimate momentum fluxes because we do not include the highest velocity channels (as discussed above). For two sources, IRAS 03282+3035 (77) and B5 IRS1 (78), we measure momentum fluxes more than a factor of 10 lower than previous estimates. We have no reason to suspect the calibration for these two maps, and they do not have particularly broad linewings (as can be seen in Fig. A.1, online). It is possible that in these flows, the momentum flux measured at greater radii in the flow is larger than in the region covered by our $2^{\prime}$ maps.

\subsection{Momentum flux vs. $L_{b o l}, M_{e n v}$ and $T_{b o l}$}

The outflow momentum flux $F_{\mathrm{CO}}$ is plotted versus core mass $M_{\text {env }}$, bolometric luminosity $L_{\mathrm{bol}}$ and bolometric temperature $T_{\text {bol }}$ in Fig. 4 (see Paper II, for the calculation of those quantities from the SEDs). The well known relationship between momentum flux and bolometric luminosity (Bontemps et al. 1996; Cabrit \& Bertout 1992) is evident despite the intrinsic scatter in the momentum flux due to uncorrected outflow inclination. This correlation is often taken as evidence that the outflow and the accretion luminosity are both being powered by the same mechanism, infall, and despite the details of the magnetic ejection/accretion process in the inner few tens of $\mathrm{AU}$ of the disk, the energy input into each remains strongly linked. The correlation is tighter towards higher $L_{\mathrm{bol}}$ and holds for both Class 0 and Class I sources (marked by red asterisks and blue open stars, respectively; see Paper II for details of classification).

Bontemps et al. (1996) found a decline of momentum flux between the Class 0 and Class I phases which has been taken as evidence that outflow power declines with age. In our sample, this is less clear cut. The mean $\log _{10}\left(F_{\mathrm{CO}} / M_{\odot} \mathrm{km} \mathrm{s}^{-1} \mathrm{yr}^{-1}\right)$ of the Class 0 population is -6.0 with a sample standard deviation of 0.6 , close to the Class I mean of -6.1 (standard deviation 0.8 ). A histogram of the momentum flux distribution for Class 0 and Class I sources (Fig. 5) shows that both of the distributions are very flat. Applying the Kolmogorov-Smirnov (K-S) test indicates a $27 \%$ probability that the two samples come from the same parent distribution and therefore there is no evidence for evolution in momentum flux between Class 0 and Class I. There is considerable scatter with objects of both classes with both low and high- $F_{\mathrm{CO}}$.

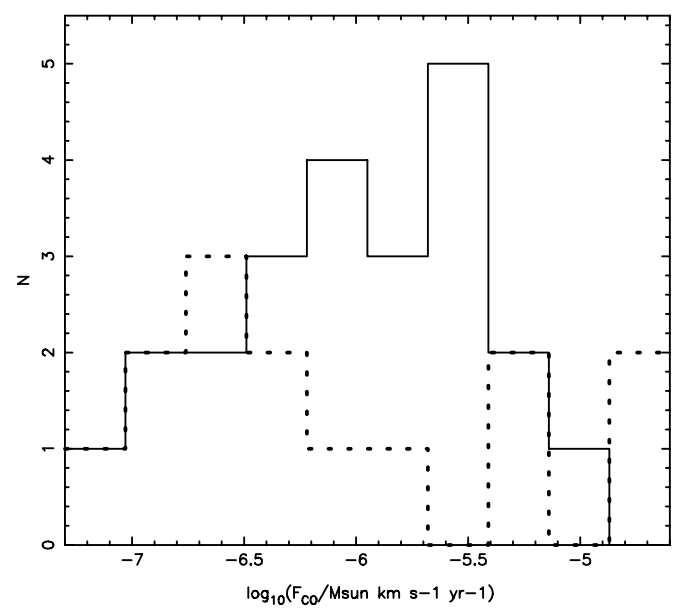

Fig. 5. Histograms of momentum flux for Class 0 (solid) and Class I (dotted) protostars.

In particular, three Class I objects- NGC 1333 SVS13 (43), NGC 1333 ASR114 (45) and NGC 1333 HH7-11 MMS4 (50)have particularly high momentum flux and four Class 0 sources b1-c (1), b1-d (4), B1 SMM5 (76) and IC 348 SMM3 (15) have low momentum flux. The third panel of Fig. 4, in which momentum flux is plotted against $T_{\text {bol }}$, shows clearly that the Class Is (on the right hand side of the plot) have more scatter in momentum flux than the Class 0s. The two high $T_{\text {bol }}$ sources are the well-known Class I sources L1455 PP9 (37), which may contain multiple protostars, and L1455 FIR1/2 (39).

All three of the high momentum flux Class I cores, NGC 1333 SSV13 (43), NGC 1333 ASR114 (45) and HH7-11 MMS4 (50) are in the confused NGC 1333 region. HH7-11 MMS4 (50) is a confused source where a major contribution to the momentum flux comes from the HH7-11 driving source SSV13. Both SSV13 and ASR114 contain multiple protostars as evidenced by radio emission (Rodriguez et al. 1997; Knee \& Sandell 2000). Can this explain their high momentum fluxes despite Class I classification? NGC 1333 SSV13 (43) is a multiple source (SSV13 and 13B) potentially consisting of a Class I source (providing the infrared-based classification) and a Class 0 source (powering the outflow). The flows from SSV13/13B are confused and the momentum flux includes contributions from both, though Knee \& Sandell (2000) attributed more momentum flux to the Class I SSV13 than the potential Class 0 counterpart SSV13B. Likewise ASR114 (45) contains multiple embedded radio sources (Rodriguez et al. 1997), and the outflows are confused by the flow from the $4^{\prime}$ distant SSV13B which is believed to drive the nearby HH 12 (Knee \& Sandell 2000), and the momentum fluxes could be overestimated by the contribution from the flow from the south. It is possible that in both these cases the high momentum flux is due to a Class 0 source, either in the same submm core or nearby - an open question which could be resolved by interferometric observations of the flows.

However, the Class 0 sources with low momentum flux b1-c (1), b1-d (4), IC 348-SMM3 (15) and B1-SMM5 (76) are less easy to explain away. Three of these sources $(1,4,76)$ also have low bolometric luminosity for their mass. Our sample, because of its submm-based sample selection, may well contain a population of low-luminosity, low- $F_{\mathrm{CO}}$ Class 0 sources which were not included in earlier studies.

There are physical reasons why outflow force may vary between sources at the same evolutionary stage. The momentum in the driving wind depends on the mass accretion rate and in 


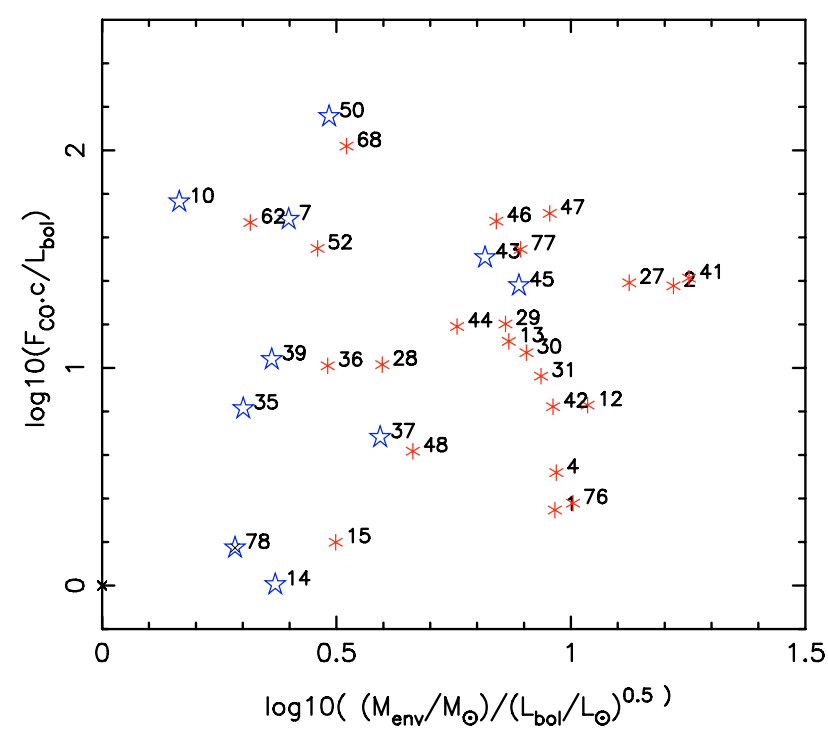

Fig. 6. $F_{\mathrm{CO}} c / L_{\mathrm{bol}}$ (dimensionless) vs. $M_{\mathrm{env}} / L_{\mathrm{bol}} 0.5$ (in $M_{\odot}$ nd $L_{\odot}$ respectively). Bontemps et al. (See also 1996, Fig. 7). Sources marked by open stars (blue) and asterisks (red) are Class I and Class 0 respectively based on the SED (Paper II).

turn on the history of the mass reservoir available for accretion. For example, this may differ between clustered and more isolated protostars (Henriksen et al. 1997), though this does not appear to explain the results in Perseus. Early Class 0 sources may not have reached their full accretion rates (Smith 2000). The low momentum flux Class 0 sources b1-c (1), b1-d (4) and B1-SMM5 (76) which also have low bolometric luminosity are likely to be early Class 0 sources which have not yet reached their full accretion power. The fraction of the wind momentum which is transferred to the molecular outflow depends on entrainment of the ambient material, which may vary in efficiency. In the competitive accretion model, mass may continue to flow onto already-accreting envelopes and boost accretion at late stages, which would lead to a natural scatter in accretion rates. Jet ejection is known to be episodic (Raga et al. 1990, 2004) but the swept-up molecular outflow smooths out any variability on timescales of $\sim 100$ years, such as those which produce jet knots.

Bontemps et al. (1996) show in their Fig. 4 a plot in which momentum flux and mass are compensated for source luminosity. In Fig. 6 we show the analogous figure for our dataset. For our sources the relationship between mass and luminosity is $L_{\text {bol }} \propto M_{\mathrm{env}}^{1.96}$ (Paper II) so the abscissa becomes $M_{\mathrm{env}} / L_{\mathrm{bol}}^{0.5}$ and the ordinate $F_{\mathrm{CO}} c / L_{\mathrm{bol}}$. The luminosity correction separates the Class I sources from the Class 0s, which tend to be more massive for the same luminosity. This separation in mass is seen in Fig. 6, with the Class 0 sources lying to the right of the diagram. It also takes out the effect of distance, though this is irrelevant for our Perseus-only sample.

The luminosity correction has to be treated with caution because it does not differentiate between luminosity and momentum flux evolution. Evidence for luminosity evolution comes from Paper II, which shows that Class 0 sources have, on average, lower luminosities at a given mass than Class I sources. Some evolutionary schemes (Smith 2000) interpret this as an increase in luminosity between the Class 0 to Class I phases. On the luminosity-corrected diagram, decreasing luminosity can be mistaken for increasing momentum flux.
Nonetheless, in the luminosity-corrected diagram, the $F_{\mathrm{CO}} c / L_{\text {bol }}$ values for the Class 0 sources are similar to their Class I counterparts, with no obvious evolution between the two stages.

There is a general trend of increasing momentum flux with $M_{\text {env }}$ (middle panel of Fig. 4) although there is more than an order of magnitude of scatter, as found for $F_{\mathrm{CO}}-L_{\mathrm{bol}}$. A physical explanation for such scatter is that the energy in the mass infall, which powers both the mass outflow and the bolometric luminosity, is dependent on the infall rate which is not set by the current mass of the core but by its past history. Hogerheijde et al. (1998) found a better correlation of $F_{\mathrm{CO}}$ with $M_{\mathrm{env}}$ than $L_{\mathrm{bol}}$, but we see no evidence for this.

In summary, there is broad agreement between our results on the momentum flux and previous studies, with similar scatter on the correlations with $L_{\mathrm{bol}}$ or $M_{\text {env }}$ despite the consistent derivation of these quantities and the single distance of our sample. A difference is the significant population of low- $F_{\mathrm{CO}}$ Class 0 sources and the small number of high- $F_{\mathrm{CO}}$ Class Is which suggest that evolution of $F_{\mathrm{CO}}$ from Class 0 to Class I is not a simple decline but that there may be variation within each class, e.g. as luminosity and outflow power increase at the beginning of the Class 0 phase.

\section{Mass loss rates and core destruction timescales}

A significant proportion of the momentum from the driving jet/wind is deposited in the protostellar core and removes matter from the core in the molecular outflow (Lada 1985; Parker et al. 1991; Fuller \& Ladd 2002; Arce \& Sargent 2006). The time it would take to clear a protostellar core of mass via the outflow is a constraint on the lifetime of the embedded protostellar phase, especially when taken together with an indication of whether the source is at the start (early Class 0 ) or end (Class I) of its embedded lifetime.

In addition to the outflow, mass is lost from the protostellar core by accretion onto the disk/star. The ratio of mass ejected in the wind/jet to mass accretion, $f$, varies significantly with different models for jet/wind launching. Observations suggest that $f \simeq 0.3$ is a good estimate (Richer et al. 2000). Although the mass loss in the jet is then less than half of the total accretion, momentum transfer to the slower molecular outflow can lead to mass loss rates in the outflow that are a factor of 10 higher than the total mass accretion rate: $\dot{M}_{\mathrm{CO}}=f \dot{M}_{\mathrm{acc}}\left(v_{\mathrm{w}} / v_{\mathrm{CO}}\right) \simeq 10 \dot{M}_{\mathrm{acc}}$, taking the jet ejection fraction $f_{a}=0.3$ and typical values for the jet and $\mathrm{CO}$ velocities of 150 and $5 \mathrm{~km} \mathrm{~s}^{-1}$ respectively, and assuming all the wind/jet momentum is deposited in the cloud. At early times when this is true, before the outflow has broken out of the cloud, then mass loss in the outflow dominates by an order of magnitude over the mass accreted onto the central object, and can be taken as a good estimate of the total mass loss rate. For more evolved flows less of the wind/jet momentum may be transferred to the $\mathrm{CO}$ and in the most evolved sources the accretion rate may begin to dominate over the mass loss in the outflow.

The outflow mass loss rate $\dot{M}$ can be calculated from the momentum flux $\dot{P}$ by dividing by the mean outflow velocity $\bar{v}$. $\bar{v}$ is a difficult quantity to estimate accurately. At low velocities it is difficult to separate outflow material from protostellar core. Any mass at high velocities can also make a large difference to $\bar{v}$ with correspondingly high uncertainties. Making an estimate using $\bar{v}=\sum_{i} I_{\mathrm{CO}}\left|v_{i}-v_{\mathrm{LSR}}\right| / \sum_{i} I_{\mathrm{CO}}$ where $I_{\mathrm{CO}}$ is the integrated spectrum over the observed inner section of the outflow, we calculate values for individual flows which range from $1-10 \mathrm{~km} \mathrm{~s}^{-1}$ 

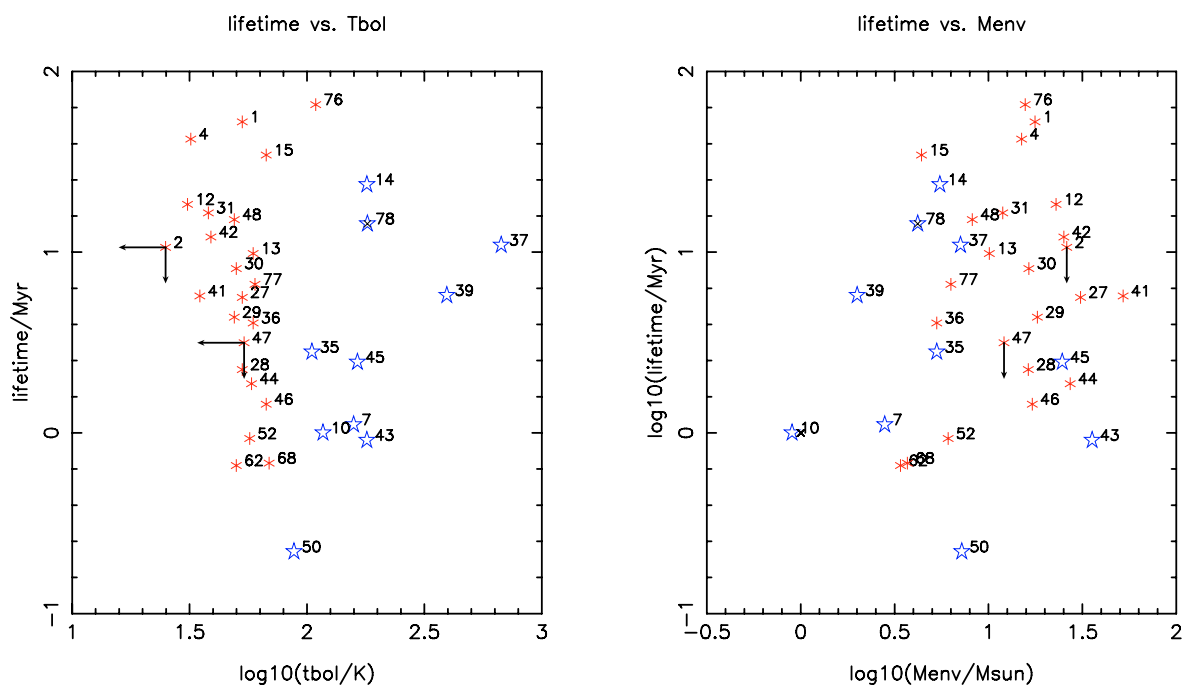

Fig. 7. Core destruction timescales at current outflow mass loss rate, vs. left bolometric temperature; right current core mass. Class I sources (Hatchell et al. 2007, based on the SED), are marked by (blue) open stars and Class 0 sources by (red) asterisks.

depending on where the integration limits - inner and outer velocity channel cutoffs - are set. We take a value of $5 \mathrm{~km} \mathrm{~s}^{-1}$ as typical, in agreement with previous estimates of $P$ and $M$ (Zhang et al. 2005; Knee \& Sandell 2000), with an uncertainty of a factor of 2 .

The resulting mass loss rates in the outflow range from $1 \times$ $10^{-7}-6 \times 10^{-5} M_{\odot} \mathrm{yr}^{-1}$, from the momentum fluxes in Table 1 , and assuming a factor of 10 to take into account inclination and optical depth into account (see Sect. 4 and Bontemps et al. 1996). These core mass loss rates are similar to those calculated by Fuller \& Ladd (2002) from the rarer CO isotopologues in molecular cores. Mass loss rates of above $10^{-4} M_{\odot}$ yr have also been found for massive flows (Zhang et al. 2005; Beuther et al. 2002; Churchwell 1997) and it is not a surprise to find rates approaching this in Perseus, which is forming stars up to spectral type B.

The destruction timescale $t_{\mathrm{d}}$ of the core at the current mass loss rate can be calculated by dividing the current core mass by the total mass loss rate, including the optical depth/inclination correction factor of 10 . These timescales are plotted in Fig. 7 and range from $0.2-60 \mathrm{Myr}$, with a mean $\bar{t}_{\mathrm{d}}=11 \mathrm{Myr}$. These destruction timescales are then lower limits to the lifetime remaining to each core, if outflows are the only source of mass loss and outflows only become weaker over time. This is believed to be true for all but the youngest Class 0 sources.

The sources with the longest lifetimes are the sources with the highest mass to momentum flux ratios. As luminosity and momentum flux are correlated (Fig. 4), these are also the sources with high mass-to-luminosity ratios and cold temperatures, a signpost of an early Class 0 source. Indeed, we find that the Class 0 sources b1-c (1), b1-d (4), IRAS 03292+3039 (76) and the newly identified protostar IC 348-SMM3 (15, Paper I, Walawender et al. 2006) have long lifetimes ( $>10 \mathrm{Myr}$ ). In all cases this is due to low momentum flux for the given core mass. Sources with particularly short destruction timescales $\sim 10^{4}$ years are IRAS 03301+3057 (7), B1 SMM11 (10) and HH7-11 MMS (43). These sources have little mass in their cores and are believed to be Class I sources near the end of the embedded phase. Thus the objects with the most extreme lifetimes are Class 0 for the longest lifetimes and Class I for the shortest lifetimes. The absence of short lifetimes for low $T_{\text {bol }}$ sources is because only high $M_{\text {env }}$ sources can produce a low $T_{\text {bol }}$, and these tend to have a long lifetime. Low-mass sources have lower optical depth in their cores and higher $T_{\text {bol }}$ for the same evolutionary state, as discussed in Paper II.

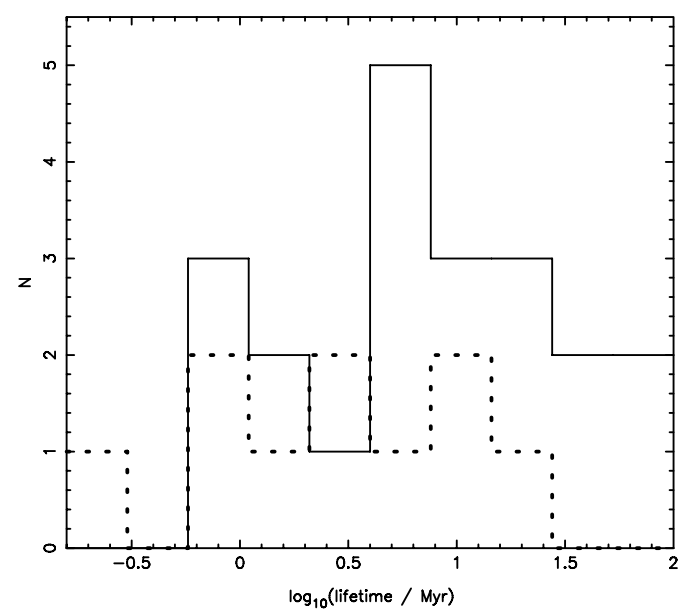

Fig. 8. Histograms of core destruction timescales for Class 0 (solid) and Class I (dotted) protostars. Sources with upper limits on the lifetime are excluded.

The mean destruction timescale for Class Is (6 Myr) is shorter than for Class 0s (14 Myr), suggesting that there might be some evolution between Class 0 and Class I, with mass declining faster than outflow force. Yet the evidence for evolution is weak: the scatter in $t_{\mathrm{d}}$ is large with both classes showing a range of destruction timescales from a few times $10^{5}$ to $\sim 10^{8}$ years at the current outflow rate, as shown in the histogram of lifetimes (Fig. 8), and a K-S test confirms a $43 \%$ probability that these populations could be drawn from the same underlying distribution.

The mean lifetime of the protostellar stage is estimated to be $1.5-4 \times 10^{5}$ years (Greene et al. 1994; Kenyon \& Hartmann 1995; Wilking et al. 1989; Kenyon et al. 1990; Hatchell et al. 2007). This is significantly shorter than our mean $t_{\mathrm{d}}$ of $10^{7}$ years for the removal of the core mass by outflows. It was also recently suggested by Arce \& Sargent (2006) that outflows may play an important role in mass loss and core evolution during the Class I phase. However, for the majority of sources, Class 0 and Class I, either we have overestimated the timescales due to systematic errors or the bulk of the mass is removed by a means other than molecular outflows. This is compounded by the fact that not all of the mass accelerated in the low velocity flow will have velocities sufficient to remove it from the gravitational field of the 
central object, so the outflows then give an upper limit on the mass loss rate to the protostellar core.

There are several factors which could introduce systematic errors in the calculation, which might result in an overestimate of the core destruction timescales. We may have overestimated the core masses: as discussed in Paper II, our core masses lie at the high end of the range consistent with the data, and could be overestimated by a factor of 4 or more - though half of this is due to the uncertain distance, which affects dust and $\mathrm{CO}$ masses equally, so again it is hard to see how this can explain more than a factor of 2 difference between protostellar lifetimes and core destruction rates by outflows. The outflow mass loss rates are calculated using several uncertain factors in the conversion of CO 3-2 intensity to mass. Firstly, there is the optical depth and inclination correction, for which we assumed a factor of 10 , and the outflow temperature, for which we assumed $50 \mathrm{~K}$, though this cannot affect the masses by more than a factor of 1.5 . The mean velocity is also uncertain to a factor 2 but the destruction timescales would only reduce if it were lower than the assumed $5 \mathrm{~km} \mathrm{~s}^{-1}$.

If protostellar cores are not destroyed by outflows and accretion, where does the mass go? A possibility is that we are detecting material that will ultimately form not single stars but small clusters, and stars will continue to form until the mass is used up, as is seen in hydrodynamical models (e.g. Bate et al. 2003). Many of the most massive cores are already known to contain multiple protostars (Sect. 4, Paper II). A further possibility is that some cores will ultimately form massive stars and the molecular cores will be destroyed by ionisation. Finally, as mass is lost from the system and the core heats up some material may become gravitationally unbound and dissipate.

\section{Summary and conclusions}

We have searched for molecular outflows towards 51 of the 103 known submm cores in Perseus listed in Paper II and based on the SCUBA (Paper I) and Bolocam (Enoch et al. 2006) surveys. The outflow sample is biased towards the higher luminosity submm cores but the fraction of cores sampled is fairly independent of mass except at the lowest masses. The main results of this study are the following:

1. We detected broad linewings from 37/51 sources surveyed. Of these, 13 cases could be due to confusion with neighbouring flows, though 9 are confirmed protostellar by Spitzer infrared detections. Only one source with a Spitzer detection has no apparent outflow.

2. The well-known correlation of momentum flux with bolometric luminosity is present, though with considerable scatter. Momentum fluxes are not significantly higher for Class 0 sources than Class I: on the basis of a K-S test, we cannot reject that the two samples are drawn from the same distribution. There are three Class I sources with apparently high momentum fluxes, possibly due to confusion with Class 0 flows, and there are several Class 0 sources with low momentum fluxes, possibly because they are very young and have not yet reached full power.

3. Core destruction timescales based on constant outflow mass loss rates and core masses from submm emission range from $10^{5}-10^{8}$ years, with no clear evolution between Class 0 and Class I sources. These timescales are longer than the estimated lifetimes for protostars of $1.5-4 \times 10^{5}$ years. There are several possible explanations for this discrepancy. These include the possibility that outflows may not be the primary mechanism which destroys the cores. Alternatively, although it appears unlikely that the core mass is overestimated due to systematic issues by a large enough factor to account for the timescale discrepancy, it is possible that we are overestimating the fraction of the core mass bound to each protostar and that the material fragments forming multiple sources.

Acknowledgements. Our thanks go to Jane Buckle, Ned Ladd and others who carried out the ${ }^{12} \mathrm{CO}$ observations, and to an anonymous referee for prompting several improvements to the paper. The James Clerk Maxwell Telescope is operated by the Joint Astronomy Centre on behalf of the Particle Physics and Astronomy Research Council of the United Kingdom, the Netherlands Organisation for Scientific Research, and the National Research Council of Canada. J.H. acknowledges support from DFG SFB 494 and the PPARC Advanced Fellowship programme. This research made use of the SIMBAD query facility of the Centre de Données Astronomiques de Strasbourg and extensive use of the Karma visualisation package developed by Richard Gooch at CSIRO (Gooch 1996), which includes an implementation of the Renzogram developed by Renzo Sancisi of the Kapteyn Institute in Groningen.

\section{Appendix A: Calculation of outflow fraction probability}

Two measured quantities are involved in calculating the fraction of protostars (outflow sources) in each mass bin. The first is the observed fraction of outflow sources in each mass bin $\mathrm{m}$, $B=b / n$ ( $n$ is the total observed sources in the bin). The second is the detection probability in that mass bin calculated from the simulation: $A=a / N$ ( $N$ is the total simulated sources in the bin). Both $a$ and $b$ are expected to follow a binomial distribution so we can easily calculate the corresponding uncertainties on $B$ and $C$ : they are just $\operatorname{Bin}(a, n, A)$ and $\operatorname{Bin}(b, n, B)$ respectively, where $\operatorname{Bin}(k, n, p)$ is the binomial distribution for $k$ successes from $n$ trials with probability $p$. With our estimates for the detected outflow fraction $A$ and detection probability $B$ we can then estimate the true protostellar fraction in the bin $C=B / A$.

Given that $a$ and $b$ are binomially distributed, we can also calculate the probability distribution for $C=A / B$ by integrating the probability space $P(A, B)$ where $B=A / C$ is satisfied.

$$
\begin{aligned}
P(C) & =\iint P(A, B) \delta(C-A / B) \mathrm{d} A \mathrm{~d} B \\
& =\int P(A) P(B=A / C) \mathrm{d} A \\
& =\int \operatorname{Bin}(a, n, A) / n \times \operatorname{Bin}(b, n, B) / N \mathrm{~d} A .
\end{aligned}
$$

This we integrated numerically for a range of values of $C$ for each mass bin and the resulting probability distributions are plotted as greyscale in Fig. 3.

\section{References}

Arce, H. G., \& Sargent, A. I. 2006, ApJ, 646, 1070

Bate, M. R., Bonnell, I. A., \& Bromm, V. 2003, MNRAS, 339, 577

Beuther, H., Schilke, P., Sridharan, T. K., et al. 2002, A\&A, 383, 892

Bontemps, S., Andre, P., Terebey, S., \& Cabrit, S. 1996, A\&A, 311, 858

Cabrit, S., \& Bertout, C. 1992, A\&A, 261, 274

Černis, K., \& Straižys, V. 2003, Baltic Astron., 12, 301

Churchwell, E. 1997, ApJ, 479, L59

de Zeeuw, P. T., Hoogerwerf, R., de Bruijne, J. H. J., Brown, A. G. A., \& Blaauw, A. 1999, AJ, 117, 354

Enoch, M. L., Young, K. E., Glenn, J., et al. 2006, ApJ, 638, 293

Evans, N. J., Allen, L. E., Blake, G. A., et al. 2003, PASP, 115, 965

Ferreira, J., \& Pelletier, G. 1995, A\&A, 295, 807 
Frerking, M. A., Langer, W. D., \& Wilson, R. W. 1982, ApJ, 262, 590

Fuller, G. A., \& Ladd, E. F. 2002, ApJ, 573, 699

Giannini, T., Nisini, B., \& Lorenzetti, D. 2001, ApJ, 555, 40

Gooch, R. 1996, in Astronomical Data Analysis Software and Systems V, ASP Conf. Ser., 101, 80

Greene, T. P., Wilking, B. A., Andre, P., Young, E. T., \& Lada, C. J. 1994, ApJ, 434, 614

Hatchell, J., Fuller, G. A., \& Ladd, E. F. 1999a, A\&A, 346, 278

Hatchell, J., Fuller, G. A., \& Ladd, E. F. 1999b, A\&A, 344, 687

Hatchell, J., Richer, J. S., Fuller, G. A., et al. 2005, A\&A, 440, 151 (Paper I)

Hatchell, J., Fuller, G., Richer, J., Harries, T., \& Ladd, E. 2007, A\&A, 468, 1009 (Paper II)

Henriksen, R., Andre, P., \& Bontemps, S. 1997, A\&A, 323, 549

Hogerheijde, M. R., van Dishoeck, E. F., Blake, G. A., \& van Langevelde, H. J. 1998, ApJ, 502, 315

Jørgensen, J. K., Johnstone, D., Kirk, H., \& Myers, P. C. 2007, ApJ, 656, 293

Kenyon, S. J., \& Hartmann, L. 1995, ApJS, 101, 117

Kenyon, S. J., Hartmann, L. W., Strom, K. M., \& Strom, S. E. 1990, AJ, 99, 869

Kirk, H., Johnstone, D., \& Di Francesco, J. 2006, ApJ, 646, 1009

Knee, L. B. G., \& Sandell, G. 2000, A\&A, 361, 671
Kregel, M., van der Kruit, P. C., \& de Blok, W. J. G. 2004, MNRAS, 352, 768 Lada, C. J. 1985, ARA\&A, 23, 267

Mangum, J. G., Bontemps, S., \& Andre, P. 1998, in 3rd Cologne-Zermatt Symposium, ed. V. Ossenkopf (Shaker-Verlag Aachen) Margulis, M., \& Lada, C. J. 1985, ApJ, 299, 925

Nisini, B., Benedettini, M., Giannini, T., et al. 2000, A\&A, 360, 297

Parker, N. D., Padman, R., \& Scott, P. F. 1991, MNRAS, 252, 442

Pelletier, G., \& Pudritz, R. E. 1992, ApJ, 394, 117

Raga, A. C., Binette, L., Canto, J., \& Calvet, N. 1990, ApJ, 364, 601

Raga, A. C., Riera, A., Masciadri, E., et al. 2004, AJ, 127, 1081

Richer, J. S., Shepherd, D. S., Cabrit, S., Bachiller, R., \& Churchwell, E. 2000, Protostars and Planets IV, 867

Rodriguez, L. F., Anglada, G., \& Curiel, S. 1997, ApJ, 480, L125

Shu, F. H., Najita, J., Ruden, S. P., \& Lizano, S. 1994, ApJ, 429, 797

Smith, M. D. 2000, Ir. Astron. J., 27, 25

Walawender, J., Bally, J., Kirk, H., et al. 2006, AJ, 132, 467

Wardle, M., \& Koenigl, A. 1993, ApJ, 410, 218

Wilking, B. A., Lada, C. J., \& Young, E. T. 1989, ApJ, 340, 823

Wilson, T. L., \& Rood, R. 1994, ARA\&A, 32, 191

Zhang, Q., Hunter, T. R., Brand, J., et al. 2005, ApJ, 625, 864 
J. Hatchell et al.: SCUBA Perseus survey. III., Online Material $p 1$

\section{Online Material}


J. Hatchell et al.: SCUBA Perseus survey. III., Online Material p 2
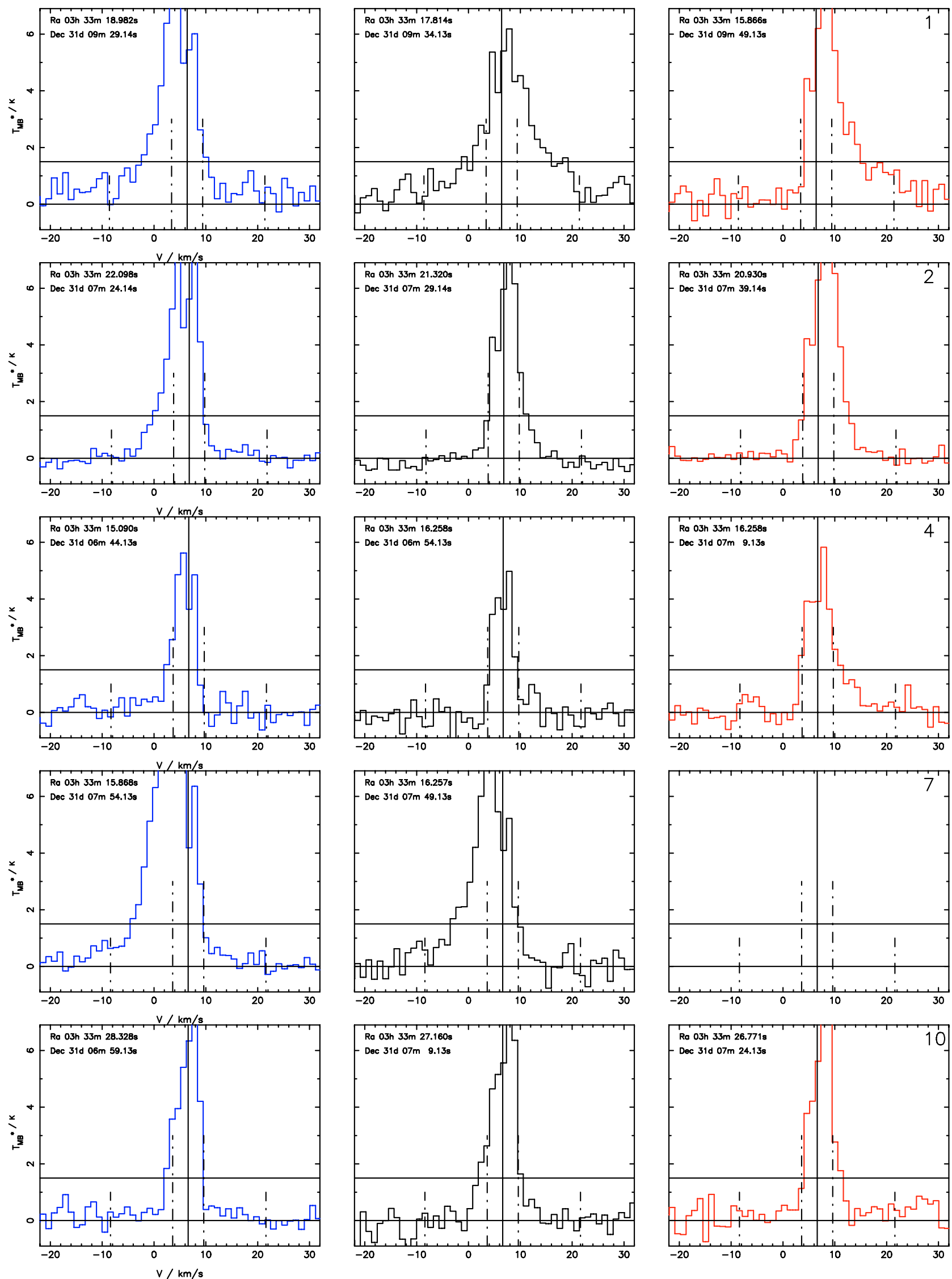

Fig. A.1. ${ }^{12} \mathrm{CO} 3-2$ spectra for outflow sources showing linewings at the $1.5 \mathrm{~K}(3 \sigma)$ level at least $3 \mathrm{~km} \mathrm{~s}^{-1}$ from the ambient velocity (marked by inner solid and vertical lines). The outer vertical lines mark the range of velocities included in the momentum flux calculation. 
J. Hatchell et al.: SCUBA Perseus survey. III., Online Material p 3
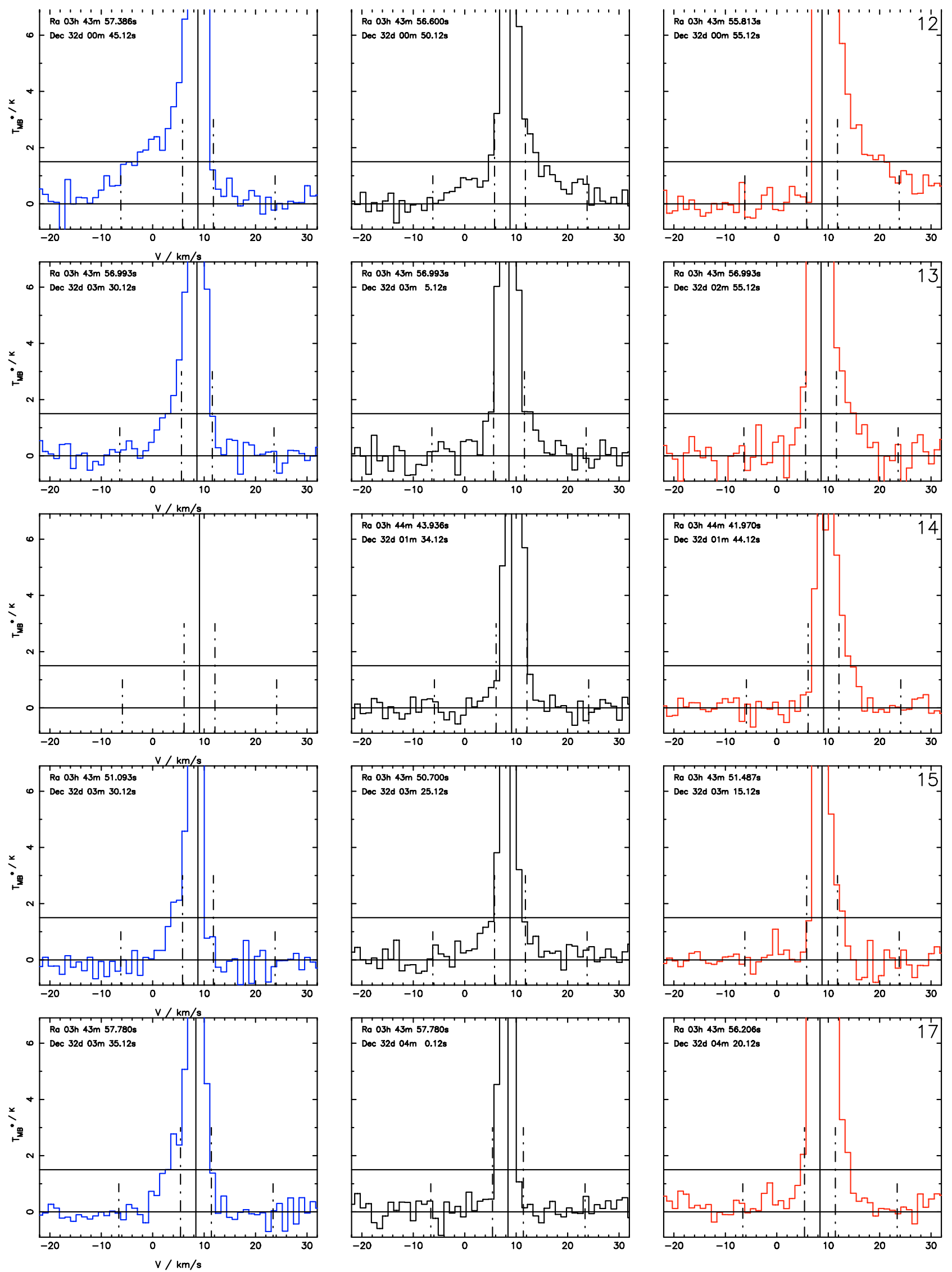

Fig. A.1. continued. 
J. Hatchell et al.: SCUBA Perseus survey. III., Online Material p 4
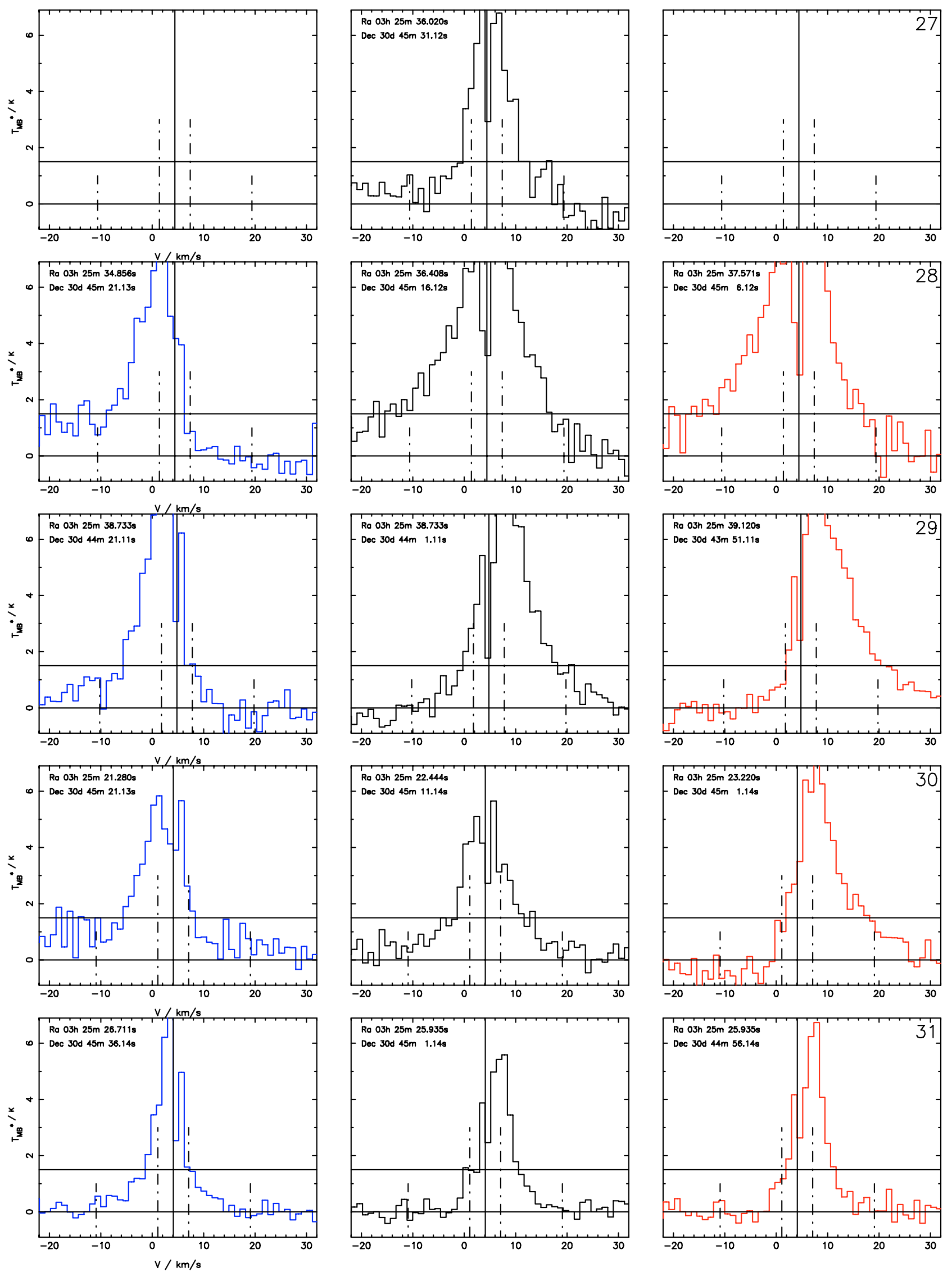

Fig. A.1. continued. 
J. Hatchell et al.: SCUBA Perseus survey. III., Online Material p 5
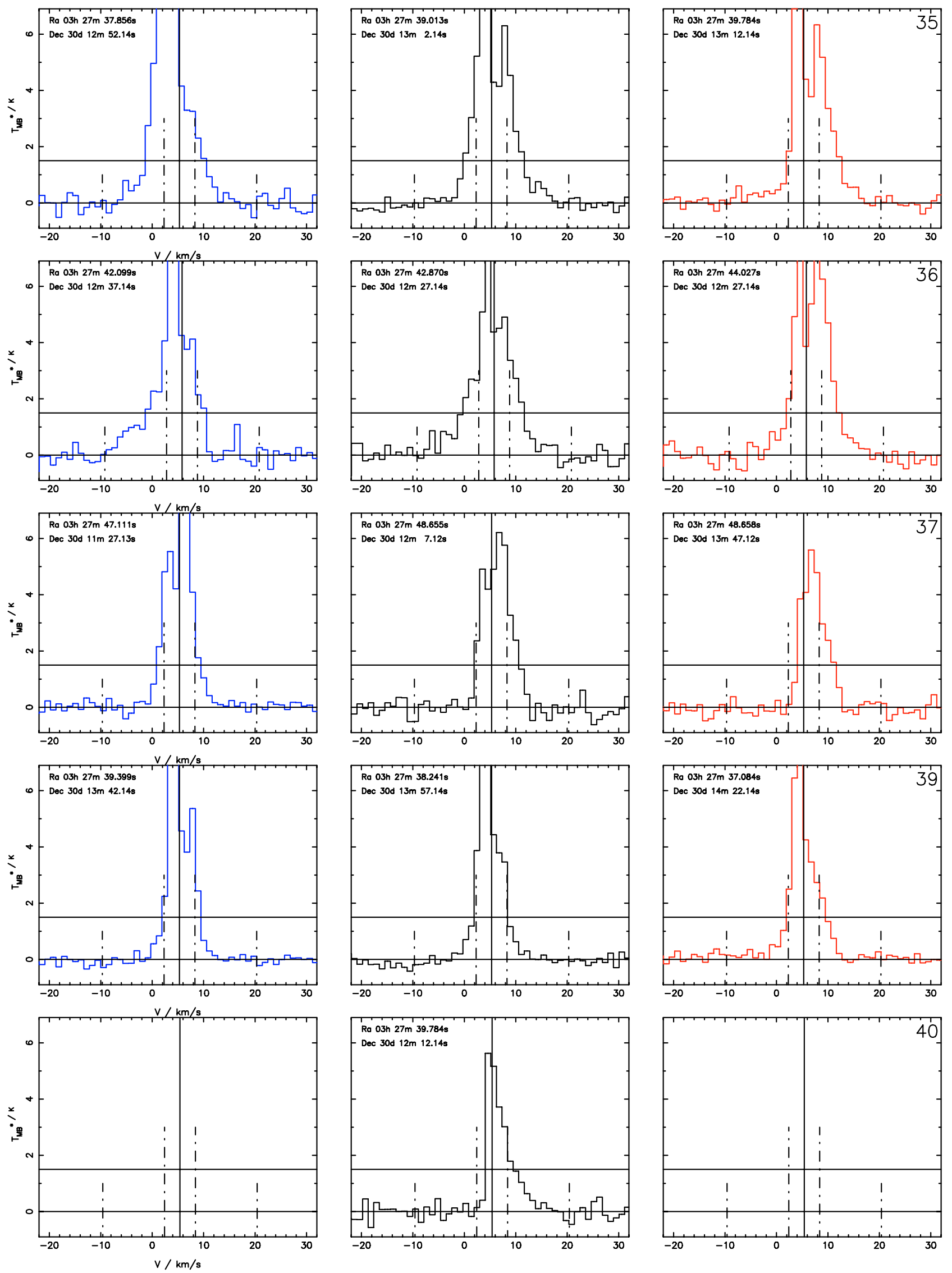

Fig. A.1. continued. 
J. Hatchell et al.: SCUBA Perseus survey. III., Online Material p 6
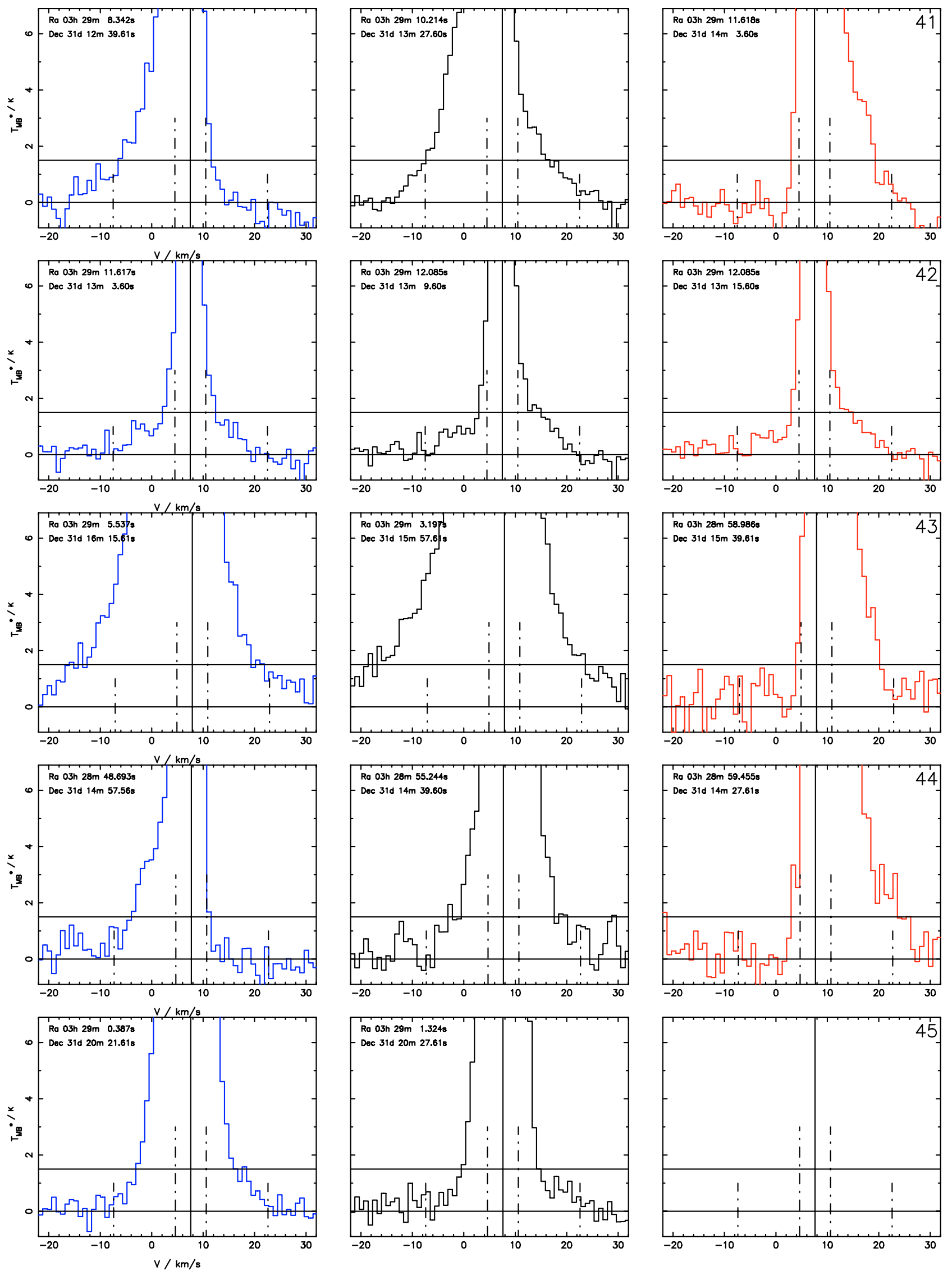

Fig. A.1. continued. 
J. Hatchell et al.: SCUBA Perseus survey. III., Online Material p 7
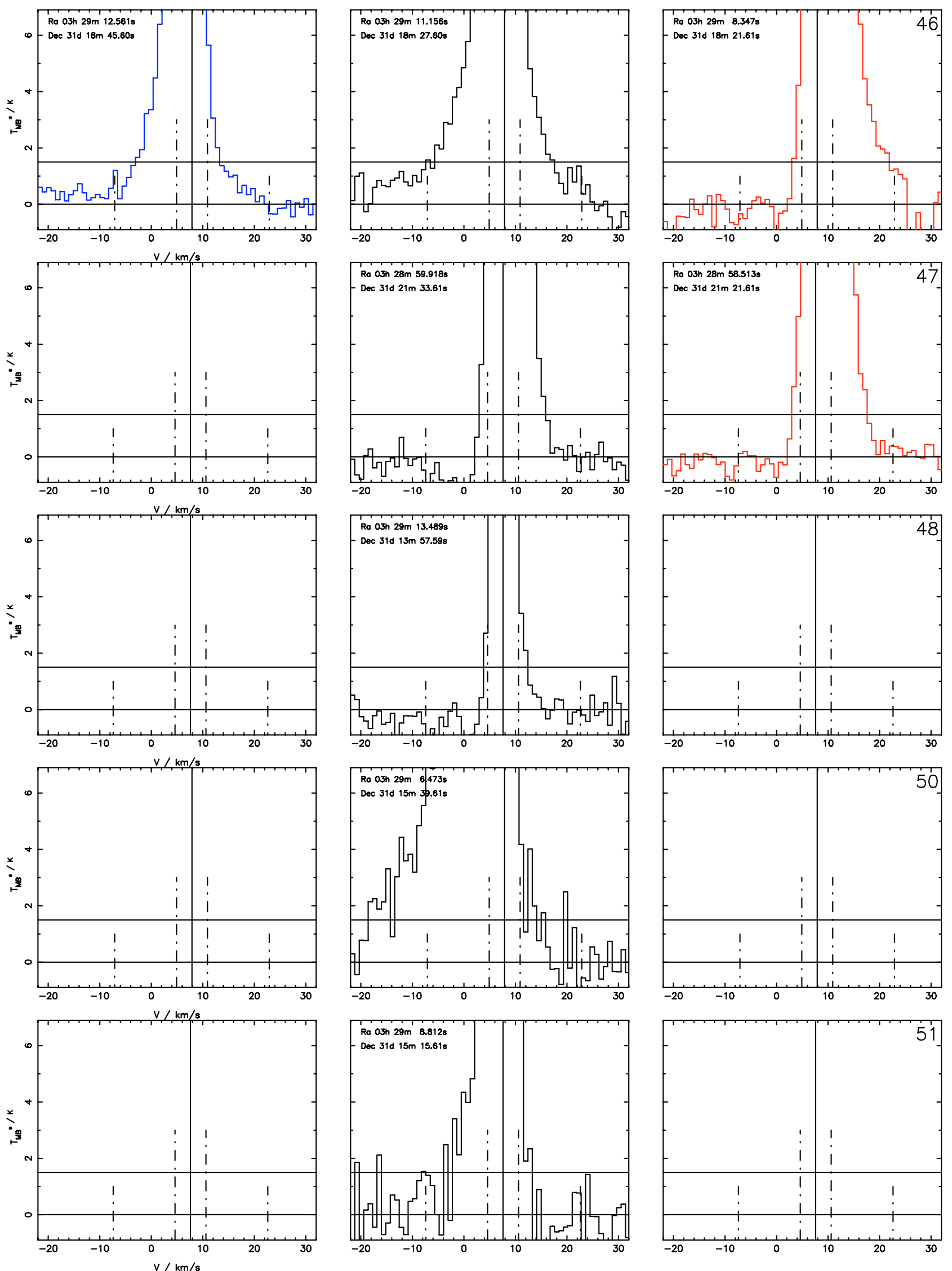

Fig. A.1. continued. 
J. Hatchell et al.: SCUBA Perseus survey. III., Online Material $p 8$
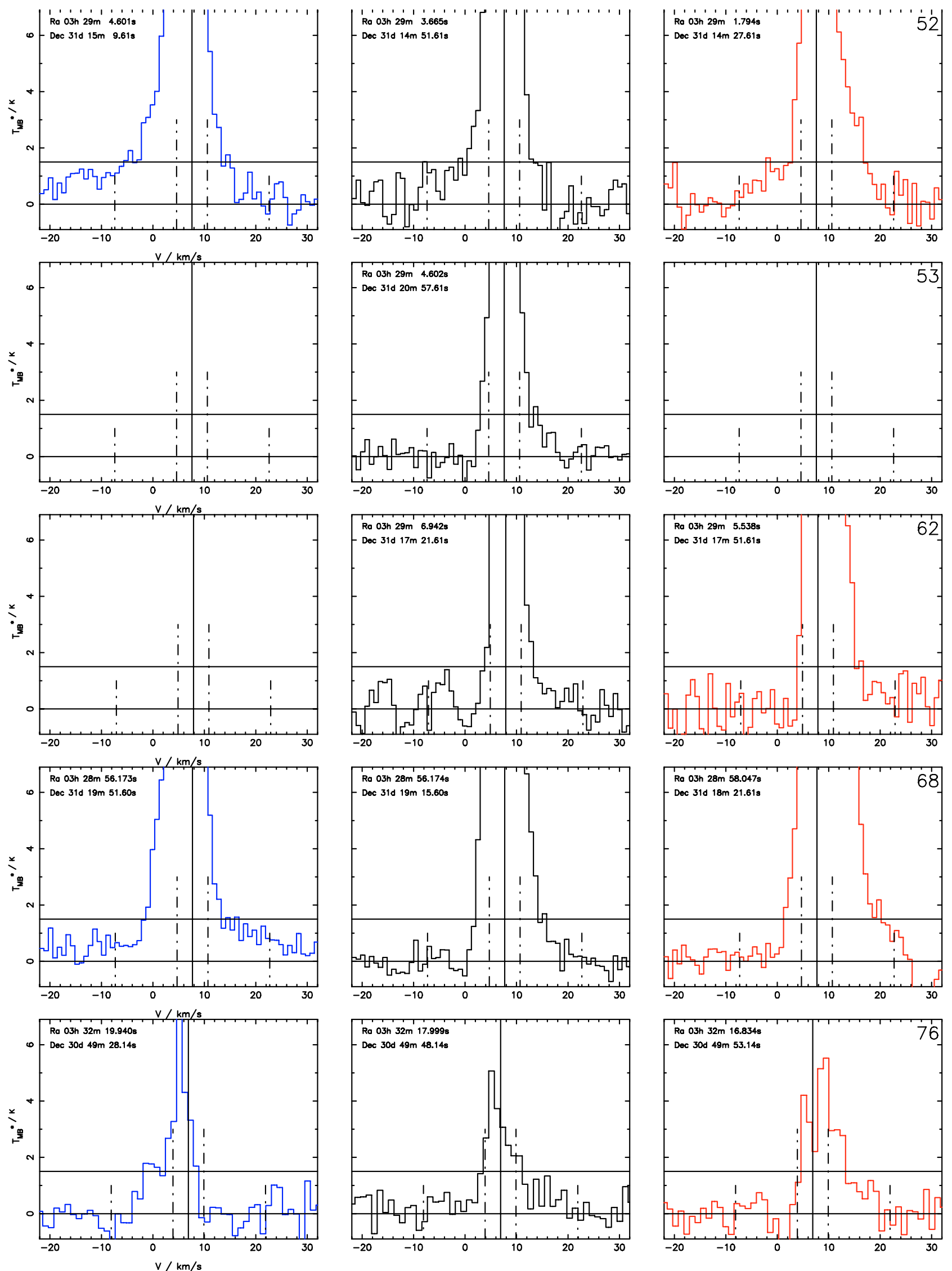

Fig. A.1. continued. 
J. Hatchell et al.: SCUBA Perseus survey. III., Online Material p 9
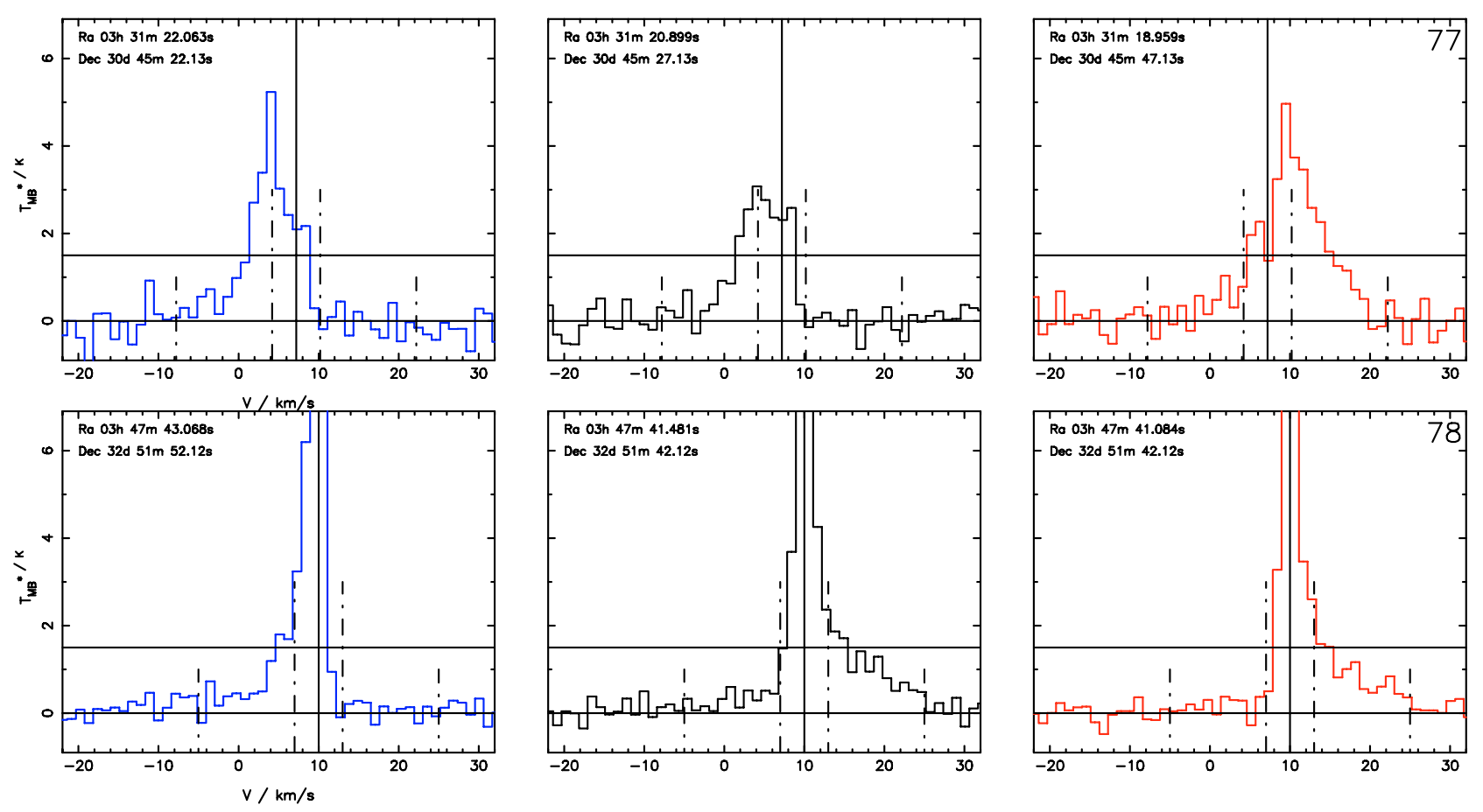

Fig. A.1. continued. 
J. Hatchell et al.: SCUBA Perseus survey. III., Online Material p 10
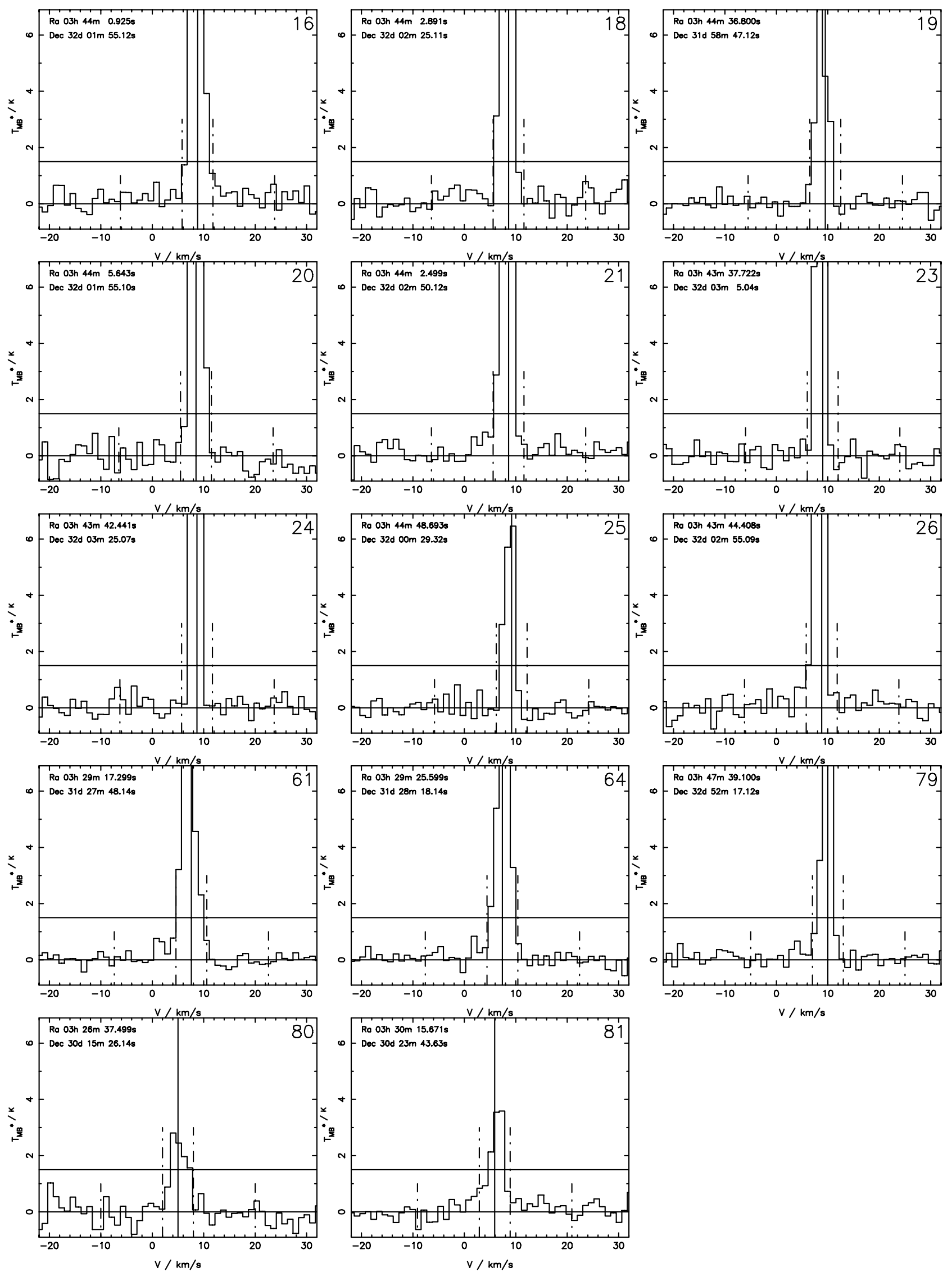

Fig. A.2. ${ }^{12} \mathrm{CO} 3-2$ spectra for sources with no outflow, showing no linewings above the $1.5 \mathrm{~K}(3 \sigma)$ level (upper horizontal solid line) at $3 \mathrm{~km} \mathrm{~s}{ }^{-1}$ from the ambient velocity (inner solid and vertical lines). 